\title{
Distance Measurements and Stellar Population Properties via Surface Brightness Fluctuations
}

\author{
Alexander Fritz \\ Gemini Observatory, 670 N. A’ohoku Place, Hilo, HI 96720, USA and \\ Istituto Nazionale di Astrofisica - Istituto di Astrofisica Spaziale e Fisica \\ Cosmica Milano, Via E. Bassini 15, 20133 Milano, Italy. \\ Email: afritz@iasf-milano.inaf.it
}

\begin{abstract}
Surface Brightness Fluctuations (SBFs) are one of the most powerful techniques to measure the distance and to constrain the unresolved stellar content of extragalactic systems. For a given bandpass, the absolute SBF magnitude $\bar{M}$ depends on the properties of the underlying stellar population. Multi-band SBFs allow scientists to probe different stages of the stellar evolution: ultraviolet and blue wavelength band SBFs are sensitive to the evolution of stars within the hot horizontal branch and post-asymptotic giant branch phases, whereas optical SBF magnitudes explore the stars within the red giant branch and horizontal branch regimes. Near- and far-infrared SBF luminosities probe the important stellar evolution stage within the asymptotic giant branch and thermally pulsating asymptotic giant branch phases. Since the first successful application by Tonry and Schneider, a multiplicity of works have used this method to expand the distance scale up to $150 \mathrm{Mpc}$ and beyond. This article gives a historical background of distance measurements, reviews the basic concepts of the SBF technique, presents a broad sample of investigations and discusses possible selection effects, biases, and limitations of the method. In particular, exciting new developments and improvements in the field of stellar population synthesis are discussed that are essential to understand the physics and properties of the populations in unresolved stellar systems. Further, promising future directions of the SBF technique are presented. With new upcoming space-based satellites such as Gaia, the SBF method will remain as one of the most important tools to derive distances to galaxies with unprecedented accuracy and to give detailed insights into the stellar content of globular clusters and galaxies.
\end{abstract}

Keywords: stars: statistics — galaxies: distances and redshifts — galaxies: elliptical and lenticular, cD galaxies: fundamental parameters — galaxies: stellar content — cosmology: distance scale

Received 2011 December 22, accepted 2012 March 26, published online 2012 May 9

\section{Introduction}

\subsection{Early Concepts}

One of the oldest and most challenging problems in astronomy is the measurement of distances to any astrophysical object on the sky. Although this task appears to be very basic in theory, it represents a major challenge in practice.

Aristarchus of Samos (310-230 BC) is probably the first astronomer to measure the distance between the Sun and Moon using trigonometry. In $265 \mathrm{BC}$ he estimated the angle between Moon-Earth-Sun at the point where the moon is exactly half full to $87^{\circ}$. Using the relation $r / R=$ $\cos 87^{\circ}$, with $r$ and $R$ being the distance to the Moon and Sun, respectively, the distance to the Sun should be 19 times the distance between Earth and Moon. Today we know that the exact angle is only sightly different $\left(89^{\circ} .85\right)$. However, not surprisingly the effect on the derived distance is huge, with the distance of the Sun from the Earth being 390 times the distance between Earth and Moon. Several other Greek astronomers, like Eratosthenes, Hipparcos, and Ptolemy, not only continued to measure the sizes of the Earth and Moon but also estimated the relative distances to the planets as far out as Saturn.

\subsection{Important Steps Towards the Cosmological Distance Ladder}

Significant advances regarding the astronomical distance scale had to await the 16th century, when Nicolaus Copernicus (1473-1543) introduced for the first time almost correct relative distances of the planets from the Sun. In 1672, John Flamsteed (1646-1719) and independently Giovanni Domenico Cassini (1625-1712) measured the distance between the Earth and the Sun to $\sim 140$ million $\mathrm{km}$ and underestimated this distance only by $7 \%$. At the same time, Sir Christopher Wren (16321723) proposed that nebulae might be distant star systems, with the light of their individual stars being blurred into a 
milky, continuous glow (the 'island universes' theory of nebulae). However, it took until the 20th century to take the next important step concerning the distance scale in establishing the distance to these (extragalactic) spiral nebulae through the discovery and application of variable Cepheid stars in the Magellanic Clouds (MCs) by Henrietta Swan Leavitt (1868-1921). Harlow Shapley (1885-1972: Shapley 1919) investigated Milky Way globular clusters (GCs) and measured their distance using pulsating Cepheid stars that were calibrated using the period-luminosity $(P-L)$ relationship for galactic Cepheids as established by Leavitt (1908). Assuming that the GCs are part of our own Galaxy, with an increased concentration towards the direction of Sagittarius, Shapley concluded that the central core of the Milky Way must be towards Sagittarius too. This correct picture, though having a wrong diameter for the Milky Way $(100 \mathrm{kpc})$ due to underestimation of the contribution of interstellar extinction (dust), yielded in 1920 to the famous great debate between Heber Doust Curtis (1872-1942) and Harlow Shapley. Heber Curtis, himself a strong supporter of the 'island universe' theory, resolved individual stars in spiral nebulae that have created novae and concluded from a comparison with novae in our own Galaxy that the novae in spiral nebulae need to be located far away. The final evidence that spiral nebulae are extragalactic systems was given later by Edwin Powell Hubble (18891953: Hubble 1926). Using new Cepheid discoveries that were calibrated to Leavitt's $P-L$ relationship for galactic Cepheids, he showed that M31 and M33 were far more distant than the Milky Way. Additional support was provided by Robert Julius Trümpler (1886-1956), who discovered the extinction to open (galactic) star clusters, and Vesto Malvin Slipher (1875-1969), who from 1912 onwards measured radial velocities of spiral galaxies with large recession velocities from the Doppler speed of spectral lines. In 1929, Hubble found a linear correlation between the apparent distances of galaxies $d$ and their recession velocities $v_{\text {pec }}$ of form $v_{\text {pec }}=H_{0} d$ (also called the Hubble law: Hubble (1929), see Section 3.3). The convincing conclusion that there indeed exists a relation between redshift and distance was given two years later using new observational data (Hubble \& Humason 1931). However, this major advance in understanding did not suggest an expansion of the Universe per se. The hypothesis of an expanding, homogeneous and isotropic Universe that is still valid today was introduced with the mathematical works of Aleksandr Friedmann (18881925), Georges Lemaître (1894-1966: Lemaître 1927), and Howard Percy Robertson (1903-1961), who all contributed independently to the classical solution of the Einstein field equations. Using the Hubble law and the assumption that the expansion of the Universe was slower at earlier times, the age of the Universe today should be $1.8 \mathrm{Gyr}$ and the Hubble constant $H_{0}=450 \mathrm{~km} \mathrm{~s}^{-1} \mathrm{Mpc}^{-1}$.

With the application of new or improved techniques in measuring distances to extragalactic objects, the extragalactic distance scale was subsequently extended to larger distances. Walter Baade (1893-1960: Baade 1944) was the first to separate the stellar populations of spiral galaxies and GCs and derived a much smaller $H_{0}$ of $250 \mathrm{~km} \mathrm{~s}^{-1} \mathrm{Mpc}^{-1}$. Allan Rex Sandage (1926-2010) discovered that the 'bright stars' seen by Hubble are actually $\mathrm{H}$ II regions that consist of star groups and clouds of ionized hydrogen. Sandage's $H_{0}$ of $\sim 75 \mathrm{~km} \mathrm{~s}^{-1} \mathrm{Mpc}^{-1}$ corresponds to an age of the Universe of $\sim 13$ Gyr. For a recent progress report on measuring the Hubble constant to a precision of $5 \%$ using the most accurate distance indicators, the reader is referred to Freedman \& Madore (2010), whereas observational probes to constrain the cosmic acceleration are presented in Riess et al. (2011) and Weinberg et al. (2012).

Figure 1 displays the extragalactic distance ladder with the most important standard candles in astronomy. The distance increases logarithmically from bottom to top, starting with our Milky Way, across the Local Group (LG) and extending towards the Local Super Cluster (LSC). Each technique represents an individual step in the distance ladder that is calibrated with a previous method of less-distant objects. The ultimate goal is a precise measurement of the Hubble constant that also gives information about the adopted galactic luminosity function and allows us to test models for dark energy (see also Section 7).

\subsection{A Brief History of Surface Brightness Fluctuations}

Early attempts in the field of Surface Brightness Fluctuations (SBFs) were conducted by Baade (1944), who resolved Population II stars in galaxies of the Local Group. Individual star fluxes in external galaxies constitute a significant part of the stellar luminosity function of red giant stars and yield, besides the distance of the galaxy, substantial information on the stellar populations. Soon thereafter, Baum \& Schwarzschild (1955) obtained observations of M31 and M32 (NGC 205) close to the limit of resolution (historically called incipient resolution') and investigated their stellar populations using the ratio of the number of resolved stars to the total integrated light (the so-called 'count-brightness ratio'). The empirical ratio of $N / L_{V \text {,tot }}$ is a useful proxy because it is sensitive to differences in the average stellar populations. The brightness was expressed in terms of visual ( $V$-band) luminosities, but is in principle applicable to all wavelengths. Other works in similar directions followed. Mould, Kristian \& Da Costa $(1983,1984)$ found similar luminosity functions for the dwarf ellipticals NGC 147 and NGC 205, whereas Pritchet \& van den Bergh (1988) and Freedman (1989) measured the tip of the red giant branch via colour-magnitude diagrams for M31 and M32, respectively. However, individual stars can only be resolved for galaxies within the Local Group, thereby limiting the power of distance measurements considerably. Unfortunately, this restriction will not significantly change with the introduction of the next generation of large telescopes; see further Section 8. Nevertheless, this problem of measuring individual star fluxes without 

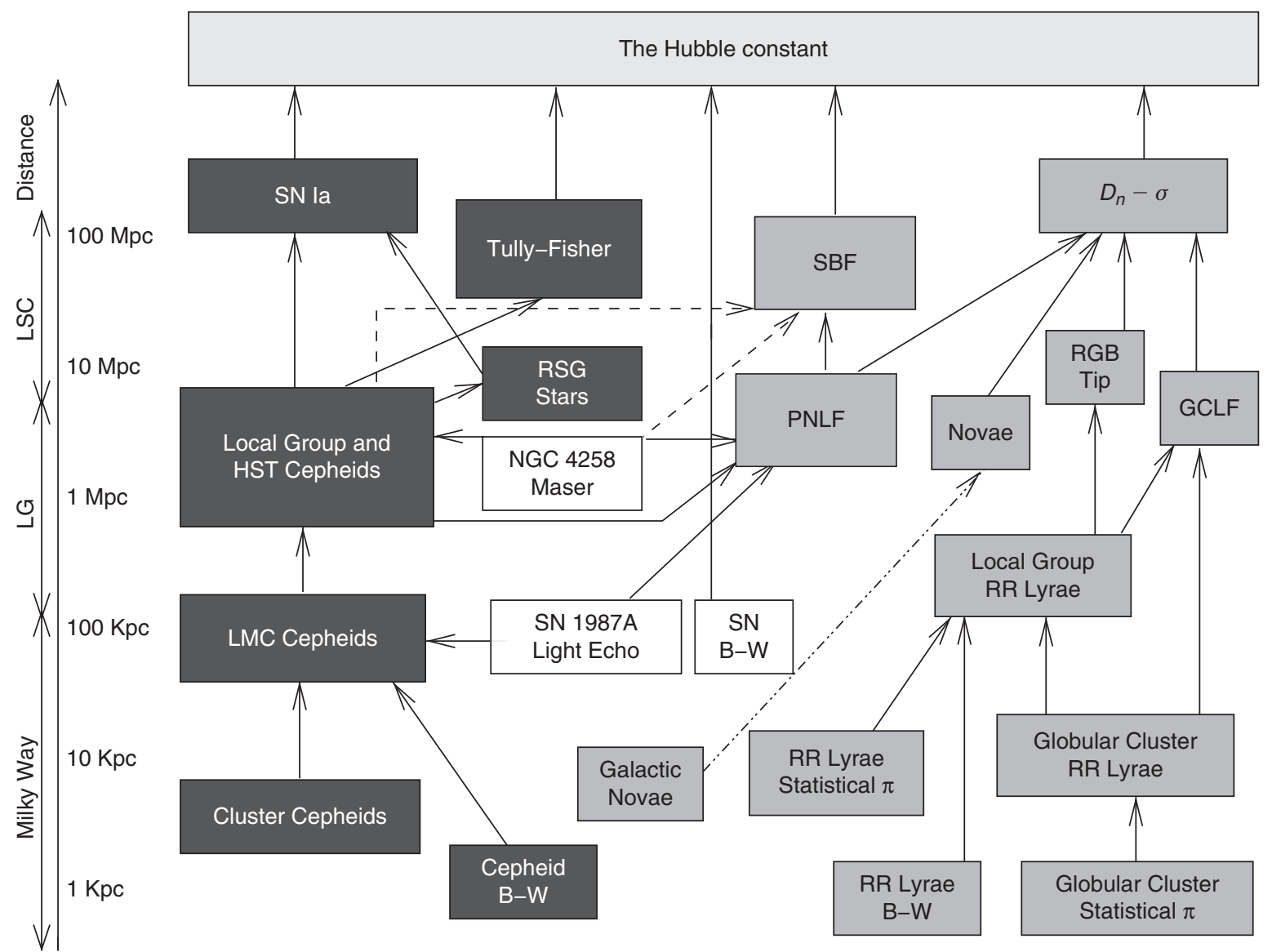

Figure 1 The extragalactic distance ladder. The dark boxes represent techniques useful in star-forming stellar systems (young Population I stars), grey-filled boxes denote methods that can be applied to quiescent stellar systems (evolved Population II stars), and open boxes give distance determinations using geometric techniques. Less-certain calibration steps are indicated as dashed lines. The distance increases logarithmically from bottom to top. Due to space constraints, the following abbreviations have been used: Baade-Wesselink method (B-W), Globular Cluster Luminosity Function (GCLF), Local Group (LG), Local Super Cluster (LSC), Planetary Nebula Luminosity Function (PNLF), Red Giant Branch (RGB), Red Super Giant (RSG), Supernova (SN), Surface Brightness Fluctuations (SBF), Parallax $(\pi)$. The figure was adapted from Ciardullo (2006).

resolving them can be bypassed if a characteristic flux of a stellar population is determined. For example, for the red populations of globular clusters this average characteristic flux can be best measured at the bluest wavelengths where the red fluctuations are smallest. An alternative way to derive fluctuation amplitudes is by using a histogram of pixel amplitudes (Baum 1986, 1990). This statistical analysis of pixel brightness variations is a variant of the 'count-brightness ratio' method and can be used to establish the upper end of the Hertzsprung-Russell diagram and through a match between simulations and real data can estimate distances as far as the Virgo galaxy cluster.

In the late 1980s, Tonry and Schneider invented a new way to quantify and measure SBFs to determine extragalactic distances (Tonry \& Schneider 1988). The basic technique of SBFs consists of spatial pixel-to-pixel brightness variations, so-called fluctuations, which arise due to the varying number of stars within the pixels in a high signal-to-noise ratio $(S / N)$ Charge-Coupled Device (CCD) image of a stellar system, like a (globular) star cluster or an early-type galaxy or spiral bulge. The details of the theory and the basics of the method are described in Section 2.
Over the past decades, several articles have discussed some aspects of the SBF technique (e.g. Tonry, Ajhar \& Luppino 1990; Tonry 1991; Jacoby et al. 1992; Tonry et al. 1997). However, there has been only one review on the subject, which concentrated mainly on the $I$ band (Blakeslee, Ajhar \& Tonry 1999).

This article presents an updated critical review of progress in the application of the SBF method. In particular, the present work focuses on new developments in this field, like the use of near-infrared (NIR) photometric bands or recent theoretical model improvements. The article is organized as follows. In Section 2, the origin and technique of SBFs is explained in detail. Section 3 gives a short overview of observational attempts in determining SBF distances from both the optical and NIR perspectives. Selection effects and biases that may alter the accuracy of the SBF method and the application of SBF as a distance indicator are presented in Section 4. In Section 5, SBFs are discussed from a theoretical point of view. Further, this section describes the recent progress obtained in stellar population synthesis modelling. An alternative way of calibrating SBF observations is presented in Section 6. Concluding remarks are drawn in 
Section 7. Finally, Section 8 outlines some forecasts for the future.

\section{The Method}

The basic idea behind the SBF technique is quite straightforward: the fluctuations in unresolved star clusters of the Milky Way and other galaxies appear, due to Poisson statistics (which is the counting statistics of discrete stars), to be clumpy and mottled, whereas in more distant objects the fluctuations appear more smooth. These spatial brightness variations, which are distancedependent in amplitude and varying for a given resolution element, are proportional to $\bar{f} \sqrt{N}$, where $\bar{f}$ denotes the mean flux per star and $N$ is the mean number of stars per pixel. Note that these values are summed over all stars of a stellar system. The mean intensity per resolution element is $N \bar{f}$; therefore the difference between the spatial variance and the observed mean results in the average flux per $\operatorname{star} \bar{f}$, which decreases inversely with the square of the distance $d^{-2} \cdot \bar{f}$ is the average flux of the underlying stellar population, weighted to its luminosity, and corresponds for evolved stellar populations roughly to the flux of a typical old giant star between spectral type $\mathrm{K}$ to $\mathrm{M}$ within the Hertzsprung-Russell diagram. If the mean absolute magnitude $\bar{M}$ is known, it is possible to determine the distance of the target object. Inversely, knowing the distance, $\bar{M}$ can be established, which itself yields information on the stellar population of a galaxy.

The average stellar luminosity $\bar{L}$ of a stellar system (star cluster or galaxy), which is summed over all stars (see Equation 2), is defined as $\bar{L}=4 \pi \bar{f} d^{2} . \bar{L}$ is weighted towards the brightest stars of a specific population. For the typical old, evolved and metal-rich stellar populations of elliptical galaxies, these are cool, luminous, red giant branch (RGB) stars $\left(3.2 \lesssim \log \left(\mathrm{L}_{\mathrm{bol}} / \mathrm{L}_{\odot}\right) \lesssim 4.2\right)$ and thermally pulsating asymptotic giant branch (TP-AGB) stars. Since (TP-) RGB stars have a red colour, the SBF magnitudes are red; ellipticals typically display $\bar{m}_{I}-$ $\bar{m}_{K} \approx 4.20 \pm 0.10$ (Jensen, Tonry \& Luppino 1998). The preferred photometric bands for SBF observations are either the red (VRI) or NIR filter bandpasses (JHK). NIR filters are preferred over optical wavelength bands, owing to two advantages in particular: (i) the SBF signal is dominated by red, luminous giant stars, and (ii) there are smaller extinction corrections due to lower dust absorption in the stellar systems. However, the KronCousins I-band supersedes the NIR bands because of its relative insensitivity to spatial variations in the stellar populations.

An observational example of the effect of spatial brightness variations is given in Figure 2. A high $S / N$ image of the galactic globular cluster M2 shows very nicely a strong lumpiness and mottling caused by the dominant evolved giant star population. In principle, this mechanism also works for galaxies, but for enhancing effects a GC was chosen. In a first step, a CCD detector measures the total flux per pixel. From this total flux the

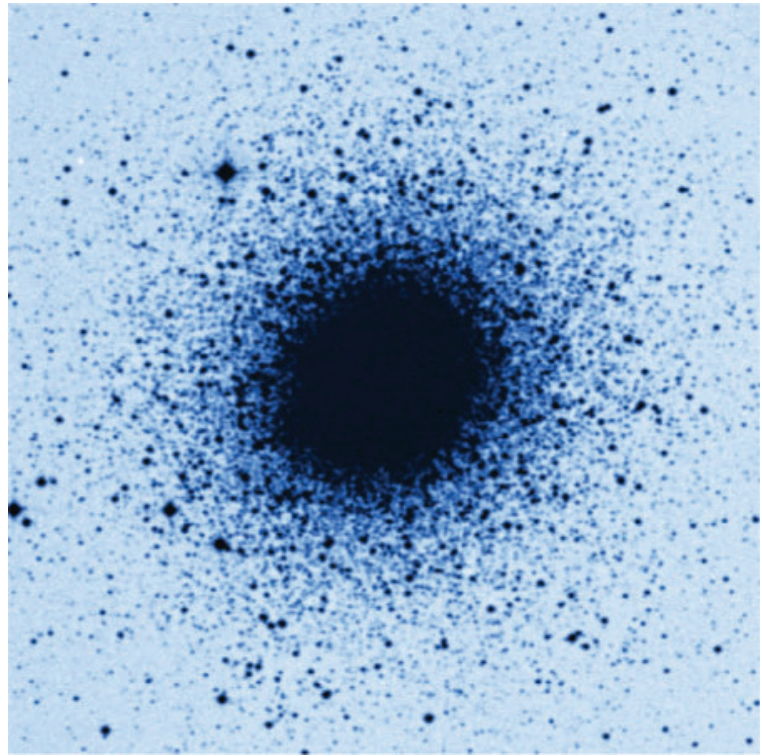

Figure 2 A high $S / N$ exposure of the galactic globular cluster M2. The composite ESO/Digitized Sky Survey 2 (DSS2) ${ }^{1}$ image clearly shows an obvious strong lumpiness and mottling that is caused by the dominant evolved giant star population. The same mechanism works for other stellar systems, such as galaxies, but is less evident because of their more complex stellar content and greater distance.

average flux per pixel (which is also known as surface brightness) and the root-mean-square (rms) variation of the flux from pixel to pixel can be derived. However, it is impossible to distinguish the two stellar systems by their average flux per pixel, because the number of stars per pixel of a resolution element increases with the distance $d^{2}$, whereas simultaneously the flux per star decreases inversely with the square of the distance $\left(1 / d^{2}\right)$. Stars cannot be resolved individually; only a characteristic (mean) flux per pixel can be established. If the number of detected photons within a resolution element is larger than the projected number of stars within this area, the fluctuations are proportional to the square root of the number of stars; i.e., the variations follow $\bar{f} \sqrt{N}$, with $\bar{f}$ being the average flux per star and $N$ the average number of stars per pixel. Thus, the variance of fluctuations $\sigma_{f}^{2}$ is derived from the square of the fluctuations from pixel to pixel as $\sigma_{f}^{2}=\bar{f}^{2} N$, where $N \bar{f}$ denotes the mean flux per pixel. The average flux per star is determined from the ratio between the fluctuation variance and the average flux per pixel as

$$
\bar{f}=\frac{\sigma_{f}^{2}}{\bar{f}_{\text {pix }}}=\frac{\bar{f}^{2} N}{\bar{f}_{\text {pix }}} .
$$

$\bar{f}$ is the average flux of the underlying stellar population, weighted to its luminosity, and corresponds for evolved stellar populations roughly to the flux of old RGB stars.

A sketch of the SBF technique is illustrated in Figure 3. Let us compare two stellar systems, a nearby galaxy (G1, left panels of Figure 3) and a second more distant galaxy

${ }^{1}$ ESO Online DSS2: http: //archive.eso.org/dss/dss 


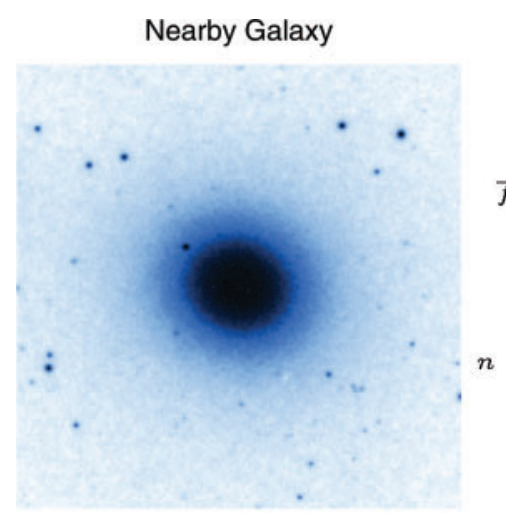

Residuals on CCD

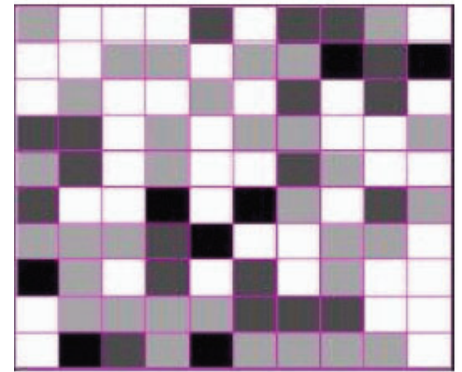

Blurred Residuals

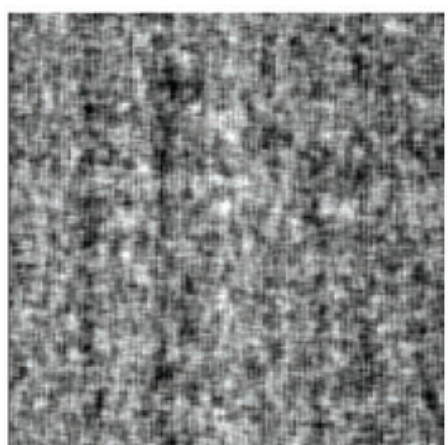

Distant Galaxy

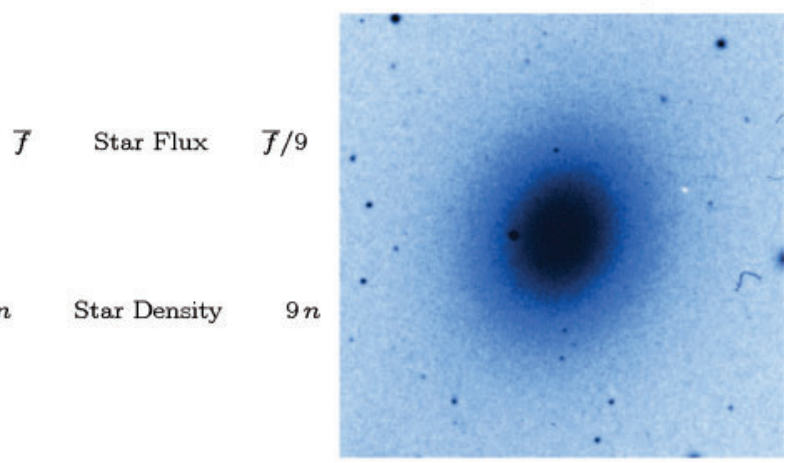

Residuals on CCD
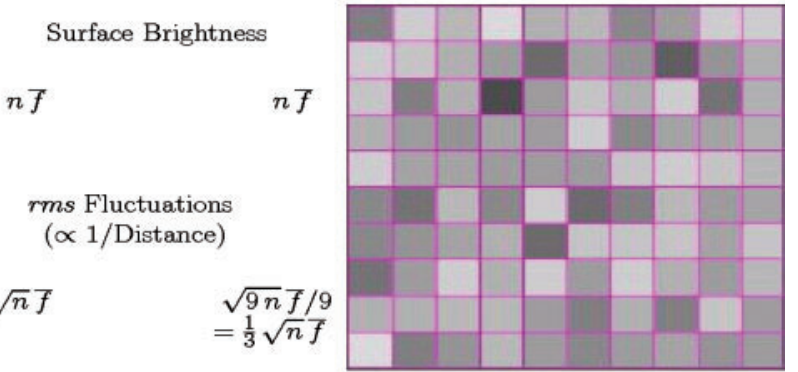

Blurred Residuals

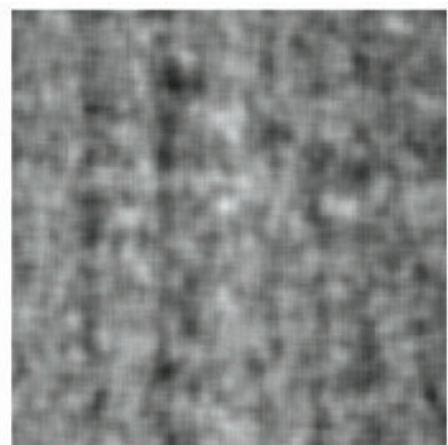

Figure 3 Sketch of the SBF method. A nearby galaxy $\left(\mathrm{G} 1\right.$, left $\left.{ }^{1}\right)$ is compared with a distant galaxy $\left(\mathrm{G} 2\right.$, right $\left.{ }^{1}\right)$ with a three times larger distance than the nearby stellar system. Note the slightly mottled structure seen in the outer parts of galaxy G1. The middle panels represent the galaxy-subtracted SBF residual view as seen on a CCD chip. One square in the image corresponds to a single CCD pixel. The bottom panels show the galaxy-subtracted SBF residuals convolved with the observed seeing to simulate the blurring caused by the Earth's atmosphere. See text for a detailed description.

(G2, right panels of Figure 3), three times as distant as the first one. Let us now assume that for the nearby G1 the average number density is 100 stars pixel $^{-1}$. The fluctuations with rms variations from pixel to pixel (rms fluctuations) are therefore $10 \%$ of the mean signal. At different regions in the galaxy, the fluctuations vary as the square root of the underlying local mean galaxy brightness. Therefore, there is a proportionality constant between the rms fluctuations and the square root of the mean surface brightness, which is directly related to the number of stars present: $\mathrm{rms} \propto \sqrt{N} \bar{f} \propto 1 / d$. If we now consider the distant galaxy, G2 has an average number density of 900 stars pixel $^{-1}$. At the same time, the star flux is decreased by a factor of 9 (the surface brightness remains constant), thus the galaxy contains only one-third of the rms fluctuations from pixel to pixel $(3.3 \%$ of the mean signal). In the second galaxy, G2, different regions in the galaxy will follow a linear relationship between the rms fluctuations and the square root of the galaxy flux. A comparison between $\mathrm{G} 1$ and $\mathrm{G} 2$ yields the surface brightness as a constant. As the proportional constant of a stellar system is inversely proportional to the distance $(1 / d)$, the constant of galaxy G2 is one-third of that for the nearby galaxy G1.

From a theoretical point of view, the SBF method relies on using the ratio of the second moment to the first moment of the stellar luminosity function (LF) of the galaxy as

$$
\bar{L} \equiv \frac{\sum_{i} n_{i} L_{i}^{2}}{\sum_{i} n_{i} L_{i}},
$$


with $n_{i}$ being the number of stars of spectral type $i$ and luminosity $L_{i}$. The mean fluctuation luminosity $\bar{L}$ depends on the stellar population and the galaxy colour itself depends on the underlying population. It is important to mention that the relation in Equation 2 is very insensitive to the uncertain faint end of the LF. Assuming a simple power-law luminosity function, the fluctuation luminosity $\bar{L}$ scales linearly with the maximum luminosity of the stars $L_{i}$.

The primary utility of SBFs as an extragalactic distance indicator will be evaluated in Section 4. Since the data collected for SBF analysis can also be used to determine the surface brightness, which provides information about the first moment of the stellar LF, the surface brightness therefore allows a measure of the stellar content solely from the integrated flux. The utility of SBFs as stellar population tools will be discussed in Section 3.4. Further, as the SBFs also depend on the second moment of the stellar LF, the SBF signal is more sensitive to the most luminous stars in a stellar system. In the case of elliptical galaxies, these stars are evolved cool giant stars. The application of SBFs as a constraint on the evolution of evolved stellar populations will be presented in Section 5.

\subsection{Types of Fluctuations}

Within a single CCD exposure of a stellar system there are a number of pixel-to-pixel fluctuations and the individual sources can basically be divided into three groups: (1) intrinsic fluctuations from the target of interest itself (e.g. a galaxy), (2) fluctuations from other objects, and (3) fluctuations caused from the instrumentation.

Intrinsic fluctuations are sources that account for additional contributions to the basic galaxy fluctuation signal and are part of the galaxy or its environment (e.g. GCs, $\mathrm{H}$ II regions, planetary nebulae, satellite systems (dwarf galaxies)). Moreover, the SBFs we are interested in and want to measure are included in this group too. Other fluctuations arise from unwanted sources (primarily foreground stars or background galaxies) that are on the same exposure as the target of interest. The third group of fluctuations originates from the instrumental set-up used (telescope and optics) and the detector itself (readout noise of the CCD camera, photon shot noise) and from cosmetic artefacts (cosmic rays, traps, defect columns, noise from the counting statistics of the flat-field exposures, or residual flattening problems after flat-field correction). Additional Poisson noise is generated from the number of detected photons in each pixel of the target itself.

Patchy (dust) obscuration results in images that are mottled or partly obscured and affects the flux of a stellar system by creating additional variance. Smooth (dust) obscuration can reduce the derived flux and the variance we seek to measure. By restricting the SBF analysis to elliptical and S0 galaxies, the problems of patchy dust obscuration are largely reduced. Moreover, these earlytype galaxies exhibit such high velocity dispersions that only dense (c)lumps endure in the fluctuation flux measurements, hence limiting possible pixel-to-pixel correlations from gravitational clumping. However, using high- $S / N$ images with sufficient resolution at blue wavelengths ( $U$ or $B$ bands) the contamination of dust can be firmly established and hence the SBF technique can be extended to early-type spiral galaxies or bulge-dominated galaxies.

There are two main factors of the fluctuations caused by the instrumentation used: the readout noise and the device photon shot noise. The variance of the readout noise is given by a relation between the inverse CCD gain $a$ (in units of electrons per analogue-to-digital unit (ADU)) and the CCD readout noise $N_{\mathrm{R}}$ (in units of electrons) as (Tonry \& Schneider 1988)

$$
\sigma_{\mathrm{R}}^{2}=\frac{N_{\mathrm{R}}^{2}}{a^{2}} .
$$

The formula of the variance of the readout noise is composed of the contributions from the inverse CCD gain $a$ and the average total signal $\bar{c}(x, y)$ at point $(x, y)$ on the CCD chip in ADU. For the mean total signal, the bias level was subtracted and the detector response and pixelto-pixel variations (flat-field) were corrected, whereas the mean sky brightness has not been removed. The variance of the photon shot noise, which is the noise due to the photon-counting statistics, is defined as

$$
\sigma_{\mathrm{P}}^{2}=\frac{\bar{c}}{a}
$$

The average total signal $\bar{c}$ is denoted by the relation $\bar{c}=\bar{g}+s$, with $\bar{g}(x, y)$ being the mean signal of the stellar system at point $(x, y)$ in ADU and $s$ being the sky flux.

All these sources of instrumental related noise are described by a white power spectrum and can therefore be separated from the intrinsic fluctuations from the stellar component of the target system, which are represented by a power spectrum of a point-spread function (PSF); see further Section 2.2. Intrinsic fluctuations produced from sources other than the target object (e.g. stars, globular clusters, faint background galaxies) are more difficult, because these sources are characterized by a similar power spectrum as the spatial luminosity fluctuations that we are interested in. Further, the variance signal of the luminosity fluctuations could be corrupted by the presence of spiral arms or star-forming regions, which would invalidate the general assumption that adjacent pixels are independent samples of the average stellar population.

\subsection{SBF Signal Measurement}

In the following, the basic procedures involved in measuring a fluctuation flux are presented. The details of the complete data-reduction steps and analysis tools are beyond the scope of this review. The interested reader is therefore referred to Tonry et al. (1990, hereafter TAL90), 
Sodemann \& Thomsen (1995), Blakeslee et al. (1999) and Fritz $(2000,2002)$.

Distance determinations using SBFs are based on two individual steps that are linked together: (i) measurement of a fluctuation flux and (ii) conversion to an absolute distance by assuming a calibrated, absolute fluctuation luminosity. There are several important facts regarding the SBF method:

- Preferred targets are dust-free systems, like elliptical (E) and S0 galaxies, spiral bulges or globular clusters.

- The total integration time must be sufficiently long enough to collect $\geq 5$ photons per source with apparent magnitude $\bar{m}$. Moreover, the PSF must be stable and uniform across the field during the collection of the observations. In particular, the telescope exposure time must exceed the point at which the photon shot noise per pixel is smaller than the intrinsic SBFs. This breaking point is given by the time at which 1 photoelectron is collected per giant star of brightness $\bar{m}$, with the integration time defined as

$$
T[\mathrm{sec}]=(S / N) 10^{0.4\left(\bar{m}-m_{1}\right)},
$$

where $S / N$ represents the required signal-to-noise ratio and $m_{1}$ denotes the magnitude for 1 detected photoelectron per second on the CCD detector. Typically, $\sim 5-10 e^{-}$for a star with $\bar{m}$ is sufficient to reach $S / N \sim$ 5-10, and an observation strategy with several dithered exposures is recommended to limit the impact of possible image gradients, fringe patterns, or CCD defects. The uncertainties in $\bar{m}$ are defined as

$$
\delta \bar{m}=0.03\left(\frac{\mathrm{FWHM}}{\operatorname{arcsec}} \times \frac{d}{1000 \mathrm{~km} \mathrm{~s}^{-1}}\right)^{2}
$$

with the first error term being the full-width-at-halfmaximum (FWHM) of the PSF and the second the distance $d$.

- The photometric $I$ band is a useful tool for distance measurements because the absolute fluctuation magnitude $\bar{M}$ is even brighter than the high sky background level in the NIR wavelength regime. The usage of this band also limits the impact of dust absorption. Moreover, for cluster galaxies there exists an empirical relationship of $\bar{M}_{I}$ with the mean galaxy colour with a small scatter ( $\sim 0.07 \mathrm{mag})$, which can be used to derive distances (see further Section 3.1 and Equation 9).

- The calibration of the zero-point of the absolute fluctuation magnitude $\bar{M}_{I}$ can be based on theoretical stellar population synthesis models (SBF acts as a primary distance indicator) or derived from an empirical relationship (SBF is used as a secondary distance indicator). The empirical calibration is based on observations of galactic GCs or LG galaxies. The methods using predictions from stellar population prescriptions and galactic GCs are expected to be consistent at least to the $10 \%$ level.
In practice, the basic procedure to measure a fluctuation flux is to perform high $S / N$ observations and data reductions in such a way as to obtain a smooth and uniform image that is based on a precise photometric calibration. To ensure a reliable calibration, a significant fraction of the observing time must be devoted to photometric standard stars. As can be seen from Equation 5, the fluctuation signal increases proportional to the exposure time and the ideal assumption is to obtain $\geq 10$ photoelectrons for a star of $\bar{m}$ in order to leave the regime of photon-counting (shot-noise) statistics and enter the regime of star-counting statistics. Therefore, the integration time depends only on the SBF magnitude $\bar{m}$ and the detector sensitivity, but is independent of the size of the target object. Hence, in the absence of a sky background all points in a stellar system $p_{i}$ are described by the same ratio of variance of fluctuations to variance of photoncounting statistics as $p(x, y) \propto \sigma_{f}^{2} / \sigma_{\mathrm{P}}^{2}+(1+s / \bar{g})$.

Once the initial data reduction (including cosmic-ray removal) and photometric calibration is complete, obvious point sources, background galaxies, any regions contaminated by CCD defects or dust are masked out and the sky background level in the outer districts of the stellar system is estimated using a $r^{1 / n}$ law profile $(n=4$ for E+S0 galaxies) plus constant offset (which allows both measurements of the mean galaxy colour and $\bar{m}$ as function of radius). In the next step, elliptical isophotes are fitted to the masked sky-subtracted galaxy image and a smooth galaxy model is constructed. This galaxy model is then subtracted from the masked image and corrected for large-scale background deviations (which affects only the low wave numbers in the image power spectrum, which are excluded from the determination of $\bar{m}$ ) to yield a residual image with various fluctuation contributions. From this residual image the Fourier power spectrum is constructed and the mean variance measured.

Before the determination of the average fluctuation variance, the contributions of fluctuations must be accounted for. Foreground stars, background galaxies, and GCs are identified and classified with an automatic photometric program to a specific completeness level (Sodemann \& Thomsen 1995; Fritz 2000, 2002). Their contribution to the fluctuation amplitude of interest $\left(P_{0}\right)$ can be described as

$$
P_{\text {fluc }}=P_{0}-P_{r},
$$

with $P_{r}$ being the residual fluctuation signal of undetected point sources of faint GCs and background galaxies. Usually the globular cluster luminosity function (GCLF) is assumed to be Gaussian and the background galaxy luminosity function to be a power law. Fortunately, even large uncertainties in $P_{r}(\sim 20 \%)$ contribute only marginally to the final error in $\bar{m}$.

In the next step, the total fluctuation amplitude $P_{0}$ is derived. The power spectrum of the masked data $P(k)$ consists of a constant $P_{0}$, multiplied by the power spectrum of the PSF $E(k)$ and a constant $P_{1}$ (from the 
white-noise component) following the relationship (TAL90)

$$
P(k)=P_{0} \times E(k)+P_{1} .
$$

It is expected that there is extra noise at very low wave numbers $(k \lesssim 25)$. However, $P_{0}$ is tightly constrained by the data across a wide range of wave numbers and therefore a precise measurement of the fluctuation flux and variance is possible. We also would like to emphasize that the ratio $\left(P_{0}-P_{r}\right) / P_{1}=P_{\text {fluc }} / P_{1}$ represents another good indicator of the $S / N$ level.

Finally, the fluctuation magnitude $\bar{m}$ can be derived as

$$
\bar{m}=-2.5 \log \left(P_{\text {fluc }} / T\right)+m_{1}
$$

Note that by using the masked residual image to create the expectation power spectrum of the PSF, $E(k)$, the contributions of the target of interest, background galaxies, GCs, and dust are all excluded, leaving the fluctuation variance $\sigma_{f}^{2}$ dependent on the mean flux per star $\bar{f}$ and the mean galaxy flux per pixel $N \bar{f}$ (see Equation 1).

\section{Observations}

\subsection{Optical SBFs}

Since the first successful prescription and quantification of SBFs as a distance indicator by Tonry \& Schneider (1988), a multiplicity of works have used this technique to determine distances to globular clusters and (groups of) galaxies in the optical wavelength regime out to $40 \mathrm{Mpc}$. After this theoretical interpretation, the method was endorsed observationally with the application to Virgo galaxies in the VRI bandpasses (TAL90). Tonry (1991) established the first empirical calibration of the absolute fluctuation magnitude $\bar{M}_{I}$ with a zero-point estimate relying solely on the M31 and M32 galaxies. A new indicator for measuring extragalactic distances was born.

In the following years the SBF method was applied to globular clusters (Ajhar \& Tonry 1994) and tested in the blue wavelength regime (Shopbell, Bland-Hawthorn \& Malin 1993; Simard \& Pritchet 1994; Sodemann \& Thomsen 1996). While the former approach gave good agreement with independent Cepheid measurements, the latter application yielded a rather poor characterisation $\left(\bar{m}_{B}-\bar{m}_{V} \approx 2.45 \pm 0.15\right)$, mainly due to the problematic different sensitivities of $\bar{M}_{B}$ and $\bar{M}_{V}$ to age and metallicity as well as additional observational limits (e.g. much fainter fluctuation magnitudes, lower $S / N$, stronger residual signal from isophotal twist and morphological distortions, dust or disk/ring-like features).

Several other studies utilised the method for individual galaxies and concentrated on refining the SBF method in the red optical bandpasses (Lorenz et al. 1993; Sodemann \& Thomsen 1995; Fritz 2000, 2002).

John Tonry and collaborators have conducted a large $I$-band SBF survey to measure the distance to $\sim 300$ nearby galaxies (Tonry et al. 1997, 2000, 2001 [hereafter
T01]). The main result of this programme was an accurate empirical calibration of the SBF magnitude using optical colours as (Tonry et al. 2000)

$\bar{M}_{I}=(-1.74 \pm 0.07)+(4.5 \pm 0.25) \times\left[(V-I)_{0}-1.15\right]$.

This calibration is based on 10 Cepheid and 44 SBF distances in 7 different galaxy groups and defined for galaxies in a colour range $0.95 \leq(V-I)_{0} \leq 1.30$. Both the zero-point and the slope of the relation are well defined (see Section 4.1 for a detailed discussion of uncertainties in the calibration of the SBF method). From ground-based $I$-band SBF measurements in spiral bulges, the zero-point in the $\bar{M}_{I}$ relation is tied directly to the Cepheid distance scale to an accuracy of 0.08 mag (excluding systematic uncertainties in the Large Magellanic Cloud (LMC) distance modulus with the $P-L$ relationship of $\pm 0.03 \mathrm{mag}$ (Freedman \& Madore 2010) or other methods, $\pm 0.16 \mathrm{mag}$ (Mould et al. 2000); see Section 4.1), corresponding to $\sim 4 \%$ in distance (Tonry et al. 2000). A comprehensive review of the $I$-band SBF distance survey can be found in Tonry et al. (1997, 2000) and Blakeslee et al. (1999).

Jensen et al. (2003) used the Hubble Space Telescope (HST) Near Infrared Camera and Multi-Object Spectrometer $(\mathrm{NICMOS})^{2} \mathrm{~F} 160 \mathrm{~W}$ distances to 65 galaxies (see Section 3.3) and established a slightly steeper slope for the $\bar{M}_{I}$ relation in the $H$ band than in the $I$ band. These measurements were tied to the Cepheid distances of the HST Key Project (KP) by Freedman et al. (2001), neglecting the -0.2 mag dex $^{-1}$ metallicity correction. The need for such a correction was ambiguous at that time and argued that there appeared to be a better agreement with the SBF zero-point predictions from simple stellar population (SSP) models when the metallicity correction was omitted. More recent works provide additional evidence of a Cepheid metallicity dependence (Sakai et al. 2004; Macri et al. 2006; Scowcroft et al. 2009) and suggest that metallicity effects cannot be neglected when deriving SBF distances. Further, there is a better understanding of the stellar population predictions for NIR SBFs (Raimondo 2009; González-Lópezlira et al. 2010; Lee, Worthey \& Blakeslee 2010), which is discussed in detail in Section 5.

With respect to the NIR calibration and the collection of high-quality HST Advanced Camera for Surveys (ACS) ${ }^{3}$ data (see Section 3.3), in recent years there has been some confusion as to whether the T01 distances derived from the ground-based I-band SBF distance survey (Tonry et al. 1997) require some correction. To overcome this discrepancy, Blakeslee et al. (2010) suggest applying a correction to distances determined using the relation in Equation 9, including both a zero-point (resulting from a revised Cepheid distance calibration of the KP (Freedman et al. 2001), which is -0.06 mag for the T01 distance moduli)

${ }^{2}$ HST/NICMOS: http : / / www.stsci. edu/hst/nicmos

${ }^{3}$ HST/ACS: http: / /www.stsci.edu/hst/acs 
and a second-order bias correction (originating from a distance bias in low-quality data of T01). Depending on the quality of the T01 data, the correction in the final T01 distance moduli $(m-M)_{\mathrm{T} 01}$ ranges between $+0.05 \mathrm{mag}$ (poorest quality) and $\sim-0.12$ mag (highest quality). In order to be consistent with the revised Cepheid scale, the corrected T01 calibration, and other works using a metallicity correction, distance moduli obtained with the Jensen et al. (2003) IR SBF calibration would need to be increased systematically by +0.10 mag.

Ground-based optical SBF observations are restricted to relatively low redshifts. Therefore, these measurements are affected by the local peculiar velocities and difficulties are experienced in deriving direct numbers for the Hubble constant $H_{0}$. In combination with other distance estimators (e.g $D_{n}-\sigma$, Tully-Fisher relation; see Section 3.3.1 for details) or space-based measurements, the SBF method provides much tighter constraints on the Hubble flow, as more distant galaxies can be considered. Using Fundamental Plane distances to $\sim 170$ elliptical galaxies, Blakeslee et al. (2002) derived $H_{0}=72 \pm$ $4($ random $) \pm 11$ (systematic) $\mathrm{km} \mathrm{s}^{-1} \mathrm{Mpc}^{-1}$.

\subsection{Near-Infrared SBFs}

There are several obvious advantages for the application of the SBF technique at longer wavelengths. The fluctuation signal is significantly stronger, $\sim 35$ times brighter in the $K$ than in the $I$ band, making the SBF signal detectable to greater distances. The seeing is typically much better in the IR than in the optical and this greatly improves the SBF amplitude, which is inversely proportional to the seeing FWHM. As the fluctuations are red compared to the globular cluster population, the resulting contrast between stellar and GC contributions to the SBF signal is higher and can be easier to separate. A drawback is that the sky background is larger in $K$. However, because of the red fluctuations, higher $S / N$ ratios can be reached in shorter exposure times than in the optical. Further, the extinction correction is a factor of 4 lower in $K^{\prime}$ than in $I$ and for old, metal-rich populations the stellar population models predict a weaker dependence of the $K$-band SBF on the integrated $(V-I)$ colour than in the $I$ band, reducing uncertainties in the colour determination of the stellar system (Worthey 1993). For this reason, the effects of age and metallicity are largely not degenerate. This fact acts in favour of stellar population studies of galaxies, but is less beneficial for simple distance calibrations.

The first feasibility studies of the IR SBF method were performed by Luppino \& Tonry (1993), Pahre \& Mould (1994), and Jensen, Luppino \& Tonry (1996) on a small set of galaxies in the Local Group and the Virgo Cluster. These works reported similar $\bar{M}_{K}$ calibrations of $\bar{M}_{K} \sim-5.65 \pm 0.20 \mathrm{mag}$. Jensen et al. (1998) extended the IR calibration to nine galaxies in the Fornax and Eridanus clusters using a wider range of $(V-I)$ colours, metallicities and additional Cepheid distance measurements. Using high $S / N$ ground-based NIR observations,
Jensen, Tonry \& Luppino (1999) pushed their groundbased $K$-band observations to measure distances to elliptical galaxies in the Hydra $\left(c z=4054 \mathrm{~km} \mathrm{~s}^{-1}\right)$ and Coma $\left(c z=7186 \mathrm{~km} \mathrm{~s}^{-1}\right)$ clusters. The authors suggested that $\bar{M}_{K}$ itself can be used as a standard candle and therefore give direct constraints on the Hubble constant $H_{0}$ (see Section 4 for details). Based on the Coma SBF distance, this work derives $H_{0}=85 \pm 11 \mathrm{~km} \mathrm{~s}^{-1} \mathrm{Mpc}^{-1}$.

\subsection{SBFs from HST}

A number of works have mined the HST archive to use galaxy observations of other programmes for SBF analyses. After the HST refurbishment in December 1993 (STS-61, HST-SM1), SBF observations have been collected from several allocated programmes through Cyles 5-7 and 15 using Wide-Field Planetary Camera 2 (WFPC2), ${ }^{4}$ ACS and NICMOS as well as a WFPC2 Guaranteed Time Observation (GTO) programme (Pahre et al. 1999). Further, new Wide Field Camera 3 (WFC3/ IR) $)^{5}$ observations are executed during Cycle 17.

\subsubsection{Optical}

Recent work on SBF distance determinations has concentrated on early-type galaxies observed with the HST. Space-based observations offer a number of advantages over ground-based measurements: higher spatial resolution $\left(\mathrm{FWHM}_{\mathrm{WFPC} 2}=0.18\right.$ arcsec, $\mathrm{FWHM}_{\mathrm{ACS}}=$ $0.098 \mathrm{arcsec}$ ), easier identification of GCs and dust due to the higher spatial resolution (reaching $\sim 2$ mag fainter systems), higher PSF stability and photometric calibration, lower sky background, no disturbing atmospheric absorption hence no extinction correction, and insensitivity to focus changes of the camera (as in the Fourier analysis only wave numbers outside the $\bar{m}$ range are affected, see Section 2.2 for details).

Using the WFPC2 on board the HST, Ajhar et al. (1997) targeted 16 galaxies in the F555W $(V)$ and F814W (I) filters. They found a steeper calibration of $\bar{M}_{I, \mathrm{~F} 814 \mathrm{~W}}=(-1.73 \pm 0.07)+(6.5 \pm 0.7)\left[(V-I)_{0}-1.15\right]$ compared to Tonry et al. (1997). However, the usage of the WFPC2 camera and the harder calibration of the broader F814W was impractical for measuring distances greater than the Coma cluster $(104.7 \mathrm{Mpc})$ because the observing time increases with the square of the distance.

Based on WFPC2 observations from a dedicated IDT/ GTO programme, Pahre et al. (1999) have investigated the distances to NGC 3379, NGC 4373, and NGC 4406. One important goal of this work was to demonstrate the capability of the WFPC2 camera (despite its sampling rate) to measure extragalactic distances using the SBF technique. The refurbishment was a success and the results for the three galaxies were in good agreement with previous measurements. For the bright group galaxy NGC 4373 in the Hydra-Centaurus supercluster, the authors

\footnotetext{
${ }^{4}$ HST/WFPC2: http: / /www. stsci.edu/hst/wfpc2

${ }^{5}$ HST/WFC3: http://www.stsci.edu/hst/wfc3
} 
Table 1. Comparison of the filter efficiency between WFPC2 and ACS filters

\begin{tabular}{lcc}
\hline Filter & $\begin{array}{c}\text { WFPC2 } \\
\%\end{array}$ & $\begin{array}{c}\text { ACS } \\
\%\end{array}$ \\
\hline F450W ${ }^{a}(U)$ & 8.5 & 36 \\
F555W $(B)$ & 11 & 37 \\
F606W $(V)$ & 14 & 44 \\
F702W $(R)$ & 14 & 42 \\
F814W $(I)$ & 10 & 42 \\
F850LP $(z)$ & 3.9 & 25 \\
\hline
\end{tabular}

${ }^{a}$ For ACS the values for $\mathrm{F} 475 \mathrm{~W}$ were adopted

${ }^{b}$ For ACS the values for F775W were adopted.

Numbers are based on the WFPC2 instrument handbook ${ }^{7}$

derive a distance of $d=39.6 \pm 2.2 \mathrm{Mpc}$ and a peculiar velocity for the galaxy of $415 \pm 300 \mathrm{~km} \mathrm{~s}^{-1}$. This peculiar velocity is about half as large as the $838 \mathrm{~km} \mathrm{~s}^{-1}$ peculiar velocity prediction using the Great Attractor model from Lynden-Bell et al. (1988) based on $D_{n}-\sigma$ observations.

Ajhar et al. (2001) measured the SBF signal to E+S0s that have Type Ia supernovae ( $\mathrm{SNe}$ Ia) and found no systematic error with distance. However, they detected a systematic offset of $\sim 0.25 \mathrm{mag}$ because of different Cepheid calibrations.

The higher quantum efficiency (a factor of 2-10 depending on the wavelength, see Table 1) and larger field of the Advanced Camera for Surveys Wide Field Channel (ACS/WFC) ${ }^{6}$ compared to the WFPC2 chip $\left(202 \times 202 \operatorname{arcsec}^{2}\right.$ versus $\left.134 \times 134 \operatorname{arcsec}^{2}\right)$ and better sampling of the PSF (factor of $\sim 2$ ) allows researchers to extend the SBF distance scale to distances greater than $10000 \mathrm{~km} \mathrm{~s}^{-1}$. In an effort to study an unbiased, almost complete sample of nearby galaxies, Tonry and collaborators have obtained WFPC 2 and ACS observations to $\sim 300$ galaxies (Tonry et al. 2000; T01). The I-band SBF survey provided accurate calibrations and measurements to 91 elliptical and lenticular $(\mathrm{E}+\mathrm{S} 0)$ galaxies and 9 spiral bulges up to $2000 \mathrm{~km} \mathrm{~s}^{-1}$, thereby building a link to other distance indicators that are sensitive to and extend well out into the Hubble flow, e.g., $D_{n}-\sigma$ (Dressler et al. 1987; Lynden-Bell et al. 1988), Tully-Fisher relation (Aaronson et al. 1989; Giovanelli et al. 1997), or SNe Ia (Sandage \& Tammann 1982; Riess et al. 2009).

More recently the SBF technique was applied to derive distances to dwarf galaxies in groups and clusters in the southern hemisphere using large ground-based telescope facilities (e.g. Jerjen et al. 2001, 2004; Dunn \& Jerjen 2006; Mieske, Hilker \& Infante 2006; Mieske et al. 2007; Mei et al. 2007; Blakeslee et al. 2009, 2010) or using HST ACS/WFC observations to nearby early-type galaxies (Cantiello et al. 2005, 2007a, b; Barber DeGraaff et al. 2007; Biscardi et al. 2008).

The first optical SBF distance out to $\sim 100 \mathrm{Mpc}$ using ACS/WFC F814W bandpass observations to shell galaxies

\footnotetext{
${ }^{6}$ ACS-WFC: www.stsci.edu/hst/acs / Detector/detectors ${ }^{7}$ HST-IHB: http: //www.stsci.edu/hst/wfpc2/documents / handbook/IHB_17.html
}

was measured by Biscardi et al. (2008). Barber DeGraaff et al. (2007) demonstrated that SBFs are also a powerful tool to determine the distance to barred lenticular galaxies. For the nearby SB0 galaxy NGC 1533 in the Dorado group, the authors derive $(m-M)=31.44 \pm 0.12 \mathrm{mag}$, corresponding to $d=19.4 \pm 1.1 \mathrm{Mpc}$. More recently Blakeslee et al. (2010) established an empirical calibration of the SBF method for the ACS/F814W filter (similar to the Johnson I band). Because of the much higher throughput, the F814W $\left(I_{814}\right)$ bandpass is more efficient and therefore to be preferred over the ACS/F850LP $\left(z_{850}\right)$ bandpass (see Table 1 for a comparison). However, the most extensive observing campaign has been performed in the ACS/WFC $\left(z_{850}\right)$ band as part of the ACS Virgo and Fornax Cluster Surveys (ACSVCS, ACSFCS). In total, distances to 135 nearby galaxies were derived, for 90 early-type galaxies in the Virgo cluster (Mei et al. 2005, 2007) and 43 galaxies in the Fornax cluster (Blakeslee et al. 2009); the latter also give a refinement of the calibration of the combined cluster samples. Moreover, the SBF distances to two dwarf galaxies in Virgo were presented in Blakeslee et al. (2010).

\subsubsection{Near-Infrared}

Using HST/WFPC2 observations to the galaxy NGC 4373 in the Hydra-Centaurus supercluster, Pahre et al. (1999) found good agreement (difference in $P_{0} \sim 2 \%$ ) with the results by Tonry et al. $(1990,1997)$, the latter using a different reduction and analysis package.

Jensen and collaborators measured the IR SBF distances to 16 galaxies located in the Leo, Virgo and Fornax clusters with $\leq 10000 \mathrm{~km} \mathrm{~s}^{-1}$ using the F160W NICMOS camera on board the HST (Jensen et al. 2001). They were not able to give tight constraints on the Hubble constant. For the Hubble constant they derived $H_{0}=72 \pm 2.3-76 \pm$ 1.3 (statistic) \pm 6 (systematic) $\mathrm{km} \mathrm{s}^{-1} \mathrm{Mpc}^{-1}, \quad$ depending on the distance of the galaxies under consideration. Because the Local Group is located in an underdense region of the Universe, the measurement is biased and therefore results in uncertainties and in a larger value of $H_{0}=76 \mathrm{~km} \mathrm{~s}^{-1} \mathrm{Mpc}^{-1}$. Soon thereafter, the team extended their efforts and established HST/NICMOS F160W SBF measurements for $65 \mathrm{E}+\mathrm{S} 0$ galaxies located in different environments (Jensen et al. 2003). Interestingly, they found evidence for intermediate-age stars in the stellar populations of the galaxies. The central bluer colours of the $\mathrm{E}+\mathrm{S} 0$ galaxies suggested younger stellar populations showing signs of recent star formation that are more metal-rich. The SBF technique is therefore an independent tool to give insights to the composite stellar populations of early-type galaxies.

Figure 4 shows the Hubble diagram for galaxies that have SBF distances. The redshift in the cosmic microwave background (CMB) frame is displayed as a function of distance for ground-based data of the I-band SBF survey, divided into different galaxy types (Tonry et al. 2001), complemented by additional high-S/N HST/ NICMOS F160W SBF measurements (Jensen et al. 


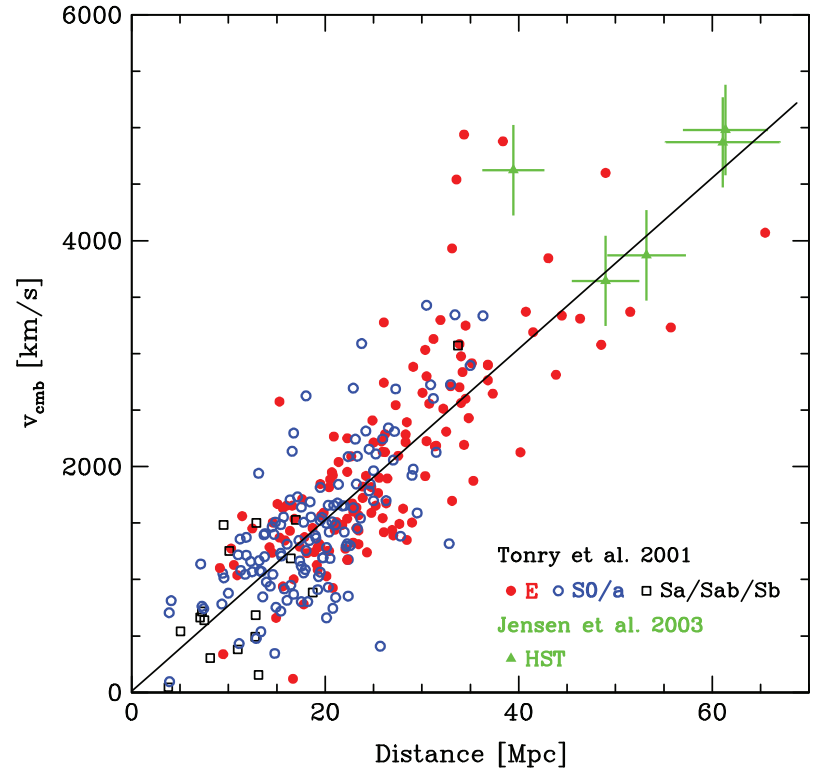

Figure 4 Hubble diagram for galaxies with SBF distances. Recession velocities in the cosmic microwave background (CMB) frame are plotted as a function of distance for ground-based data (Tonry et al. 2001, circles and squares) and HST/NICMOS measurements (Jensen et al. 2003, triangles). The line has a slope of $75 \mathrm{~km} \mathrm{~s}^{-1} \mathrm{Mpc}^{-1}$. Typical $1 \sigma$ uncertainties are indicated for the HST data. For clarity, error bars are omitted for the ground-based data. The very deviant points near $39 \mathrm{Mpc}$ are galaxies with high peculiar velocities belonging to the Cen 45 cluster of galaxies.

2003). All redshifts were corrected for the peculiar velocity of the Local Group of galaxies with respect to the CMB. The expansion of the Universe is clearly apparent and a linear, unconstrained fit to the data gives a Hubble ratio of $75 \pm 4$ (stat) \pm 7 (sys) $\mathrm{km} \mathrm{s}^{-1} \mathrm{Mpc}^{-1}$. Local peculiar velocities are the origin for scatter in the Hubble ratio at distances of $40 \mathrm{Mpc}$, but the majority of data at this distance are pretty well described by the derived Hubble value. Part of the scatter in the SBF Hubble diagram is due to contributions of external sources (e.g. differences in the stellar populations), but does not solely rely on uncertainties in the distances. In the next few years, the SBF Hubble diagram will be better defined at distances reaching out into the Hubble flow $(d>40 \mathrm{Mpc})$ and likely be extended up to $100 \mathrm{Mpc}$ using high-quality measurements of the WFC3/IR instrument on board the HST.

\subsection{Stellar Population Gradients}

Early-type galaxies are well known to show radial colour gradients due to variations in their stellar population properties (e.g. de Vaucouleurs 1961; Peletier et al. 1990). The first SBF gradients were discovered in ground-based observations for individual galaxies (Tonry 1991; Sodemann \& Thomsen 1995, 1996; Jensen et al. 1996; Fritz 2000, 2002).

Using ACS/WFC observations, Cantiello and collaborators have investigated the SBF gradients of a larger sample of nearby galaxies (Cantiello et al. 2005, 2007a). Examples of SBF colour gradients are shown in Figure 5.

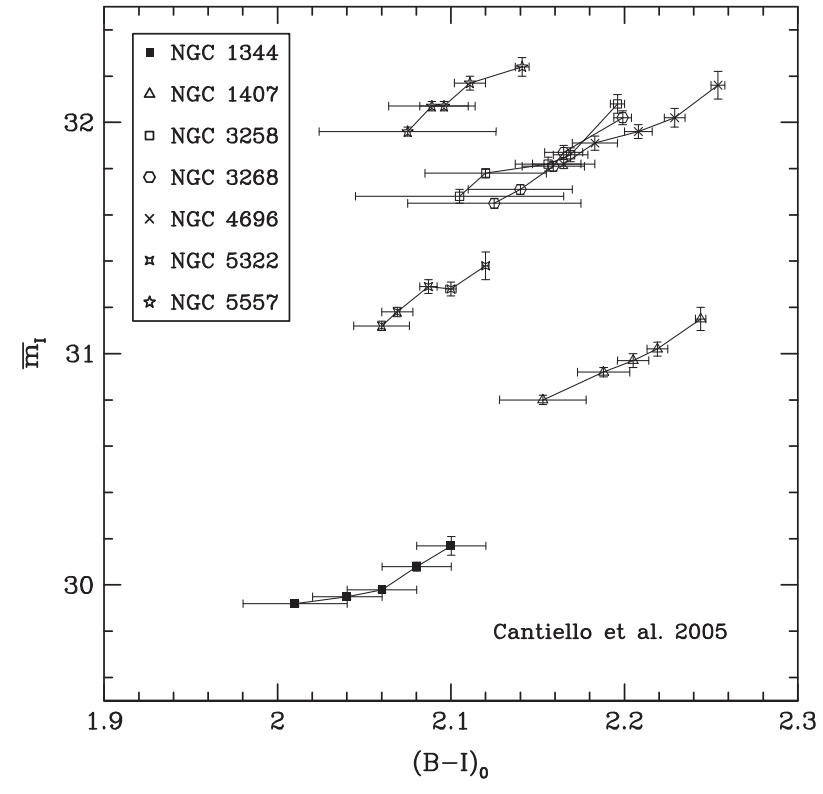

Figure 5 Apparent $I$-band SBF magnitudes in a series of concentric annuli plotted as a function of $(B-I)_{0}$ galaxy colour (Cantiello et al. 2005). With increasing radial distance from the galaxy centre, the mean galaxy colour becomes bluer and $\bar{m}_{I}$ brighter. The vertical offsets are due to the different distances of the galaxies.

The size of the slope between the intrinsic internal galaxy SBFs $\left(\bar{M}_{I}\right)$ and the galaxy colour $\left((B-I)_{0}\right)$ for multiple annuli within a galaxy allows one to disentangle whether the gradients are due to age or metallicity variations. For the majority of galaxies in Figure 5, the gradients are metallicity-driven (e.g. NGC 3258), whereas for a few cases the detection of a shallower slope suggests an age gradient which is equally important or more so (e.g. NGC 1344). Galaxies with age gradients indicate a bluer, younger stellar population at larger galaxy radii and some evidence for recent merging activity or accretion events. More studies on this topic involving larger samples would allow scientists to draw further conclusions on the origin of SBF gradients.

For the foreseeable future, the WFC3/IR camera on board $H S T$ offers a powerful new possibility of investigating the internal SBF gradients in the NIR (see also Section 8). It would be extremely exciting to compare the optical gradients with their NIR counterparts. In the NIR, pure age or metallicity variations affect the SBF magnitude dependence on galaxy colour very differently. More comprehensive studies of SBF gradients, in particular in the NIR, might allow us to disentangle the age/metallicity degeneracy in elliptical galaxies and therefore provide new insights into their different evolutionary histories.

\section{SBFs as a Standard Candle}

To derive accurate distances with the SBF technique, it is necessary that the pixel-to-pixel fluctuations are dominated by the Poisson statistics of the stars rather than by photon shot-noise statistics or other detector artefacts (Tonry \& Schneider 1988). To fulfil this constraint, one 
needs to acquire high $S / N$ images (see Section 2.2). Furthermore, other fluctuations that originate from unwanted contributions $\left(P_{r}\right)$ such as GCs, foreground and/or background galaxies, need to be removed as well (Blakeslee \& Tonry 1995; Jensen et al. 1999). In particular, for distance moduli $(m-M)>32$, the fluctuations from external sources are at least of the same order of magnitude as (or even higher than) the SBF fluctuations themselves and therefore need to be carefully removed before estimating distances (Jensen et al. 1998). This is even valid for the NIR bands, although one might expect the relative contributions to the fluctuations from GCs to be smaller, as stellar SBFs are much redder than (those of) GCs. Since the background is higher in the NIR and the sampling and sensitivity to faint point sources is decreased, this effect cancels out for the total fluctuation power (contribution of background galaxies is reduced by a factor of $\sim 10$ ).

There are two main requirements for using the SBF technique as a distance indicator: (i) the bright end of the LF among galaxies of different type (ellipticals, S0s and spiral bulges) is universal, or (ii) the variations in the LF from stellar system to system can be detected and corrected for, so that $\bar{M}$ remains calibrated at high precision, hence a standard candle (see also Section 2 and Equation 2).

Several studies have proved that absolute fluctuation magnitudes in both $I$ and $K$ bands of elliptical galaxies are correlated with the internal $(V-I)_{0}$ galaxy colour (TAL90; Luppino \& Tonry 1993; Ajhar et al. 1997; Tonry et al. 1997; Jensen et al. 1998; Mei et al. 2001; Liu, Graham \& Charlot 2002; Liu, Charlot \& Graham 2000). As a consequence the empirical calibration can be considered as universal and therefore as a standard candle. The linear relationship between absolute fluctuation magnitude and galaxy colour has been calibrated to Cepheid distance measurements in the Virgo Cluster and the Local Group (see Section 3.1 above). The intrinsic scatter in the Cepheid zero-point calibration is $\sim 0.05-0.09 \mathrm{mag}$, whereas the stellar population models (Worthey 1993) suggest an uncertainty in the $\bar{M}_{I}-(V-I)_{0}$ relation of $<0.11 \mathrm{mag}$. The exact scatter in the theory depends on the composition and the variations among the stellar populations.

Jensen et al. (1999) compared their $K$-band data to the theoretical predictions by Worthey (1993) and concluded that their fluctuation magnitudes $\bar{M}_{K}$ are almost constant in the colour range $1.10 \leq(V-I)_{0} \leq 1.30$. This implies that the $K$-band SBF itself is a good standard candle with a median absolute magnitude of $\bar{M}_{K}=-5.57 \pm 0.19$. The average scatter in $\bar{M}_{K}$ is only $0.06 \mathrm{mag}$, but the models with solar metallicity suggest brighter absolute magnitudes of $\bar{M}_{K s}=-5.84 \pm 0.04$ but a slope $(3.6 \pm 0.8)$ that is in agreement with the optical calibration (Liu et al. 2002). From this comparison it is clear that more highquality observational data are needed and a more careful description of the stellar populations has to be obtained from a theoretical point of view. A more detailed discussion on this issue with the presentation of new stellar population models is given in Section 5.

\subsection{Caveats for Distance Determinations}

As we have shown, SBF magnitudes represent a universal standard candle and hence a precise distance indicator. However, there are several possible problems and uncertainties that could affect accurate distance measurements.

\subsubsection{Target Selection}

The preferred targets for SBF measurements are dynamically hot stellar systems, either GCs or elliptical galaxies. There have been attempts to measure distances to disky S0 galaxies (NGC 1375: Lorenz et al. 1993) or edge-on spiral bulges (NGC 4565: Simard \& Pritchet 1994; NGC 3115: T01). However, apart from differences in the analysis techniques, Cepheid stars are only found in the dusty disks of spiral galaxies. Stellar systems with multiple components in the light profile (bulge, disk, bar, rings) are difficult to model for the SBF method, as the stars of the disk need to be correlated to the place where the SBF signal was measured. Hence, the SBF technique is mostly limited to the dust-free stellar systems of elliptical galaxies. An alternative approach is to go to space. Thanks to the superb resolution of HST compared to ground-based telescopes, different components and dust features can be detected, modelled, and subtracted much better and the SBF technique extended to S0 galaxies and early-type spiral bulges (Jensen et al. 2003). This approach appears to be promising, as Cepheids are most common and have a higher abundance in the outer disks of spiral galaxies, which are usually difficult to analyse with the SBF technique.

\subsubsection{PSF Modelling}

A precise stellar template is crucial for measuring the exact SBF amplitude. All modifications of the power spectrum of the stellar template translate directly to the power spectrum of the fluctuations. For example, a mismatch of $3 \%$ in the normalization of the stellar power spectrum results in an error of $0.03 \mathrm{mag}$ in the fluctuation magnitude $\bar{m}$. Usually, having a good stellar template as PSF is not a major problem, as there are stars located close to or in the surrounding field of the target galaxy. In the NIR, however, this issue is more prominent as the stars must be detectable and separated from the much higher sky background. For HST there is the possibility of constructing synthetic PSF templates to account for the PSF variations and distortion effects on the pixelsensitive WFPC2, ACS, or NICMOS chips (e.g. Fritz, Böhm \& Ziegler 2009a; Fritz et al. 2009b). In general, for ground-based observations the mismatch between real and synthetic PSFs is small (see Fritz 2002), but when real observed PSFs are available these are to be preferred.

At the present time, the real limiting factor of $(\mathrm{N}) \mathrm{IR}$ detectors is the small field of view relative to their optical counterparts. As a consequence, fewer bright field stars are available for calibration purposes that are uncontaminated by galaxy or companion light. 


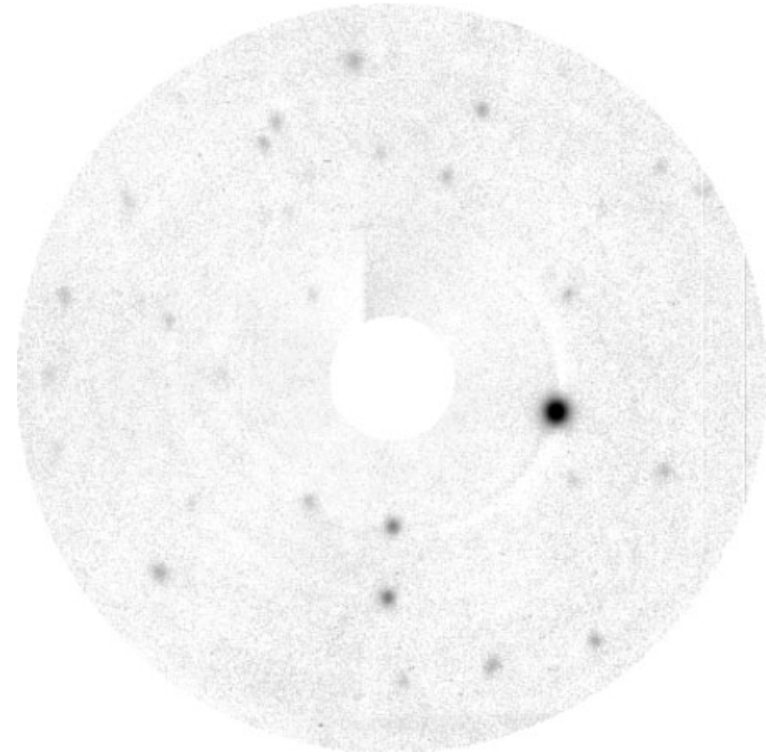

Figure 6 Masked (galaxy-subtracted) residual image of NGC 3379 (adapted from the investigation of Fritz 2002). The unsaturated bright star right of the centre of the galaxy was adopted as a PSF template. Sources in this image consist of GCs, background galaxies and SBFs. From the normalized luminosity fluctuations, a characteristic flux-weighted mean flux of $\bar{f}=3.37 \mathrm{ADU} \mathrm{star}^{-1}(300 \mathrm{~s})^{-1}$ was measured, which corresponds to a weighted mean fluctuation magnitude of $\bar{m}=28.61 \pm 0.04 \mathrm{mag}$ (Fritz 2002).

\subsubsection{Background Sources}

Ground-based SBF measurements have a crucial limiting factor: the detection, modelling and careful removal of faint background sources and globular clusters. The total fluctuation amplitude $\left(P_{0}\right)$ must be dominated by the SBF signal, hence the residual fluctuation of undetected faint GCs and background galaxies $\left(P_{r}\right)$ needs to be smaller than the SBF fluctuations. This is illustrated in Figure 6 . The superior resolution of space-based observations offers the great advantage of much more accurate detection and sensitivity to unwanted sources. Further, in the IR the sky background of NICMOS HST observations is more than a factor of 100 lower compared to IR observations from ground-based telescopes.

\subsubsection{The Good, the Bad, and the Ugly}

Considering the challenge involved in deriving a reliable estimate of the fluctuation magnitude $\bar{m}$, the measurement of the galaxy colour should represent an easy task. Instead, however, the linear correlation of $\bar{M}_{I} \propto$ $4.5(V-I)$ makes an accurate colour determination essential. Internal colour gradients in a galaxy introduce complexity and therefore cause difficulties when deriving the colour of a stellar system. Both $\bar{m}$ and $(V-I)$ need to be measured over the same region in the galaxy, which is tricky if the galaxy has multiple components of bulge plus disk and $\bar{m}$ is established in the centre, whereas $(V-I)$ is obtained from the outer regions of the disk.

For optical colours the effects of age and metallicity are largely degenerate. Therefore, going to the IR and looking for a NIR colour calibration would be greatly beneficial as it would allow one to quantify differences in the stellar populations that alter the light-averaged age and metallicity of a stellar system. A few observational (Jensen et al. 2003) and theoretical approaches (Blakeslee et al. 2001a; Lee et al. 2010) in this direction have been conducted. However, more observations and improved model descriptions would finally provide an accurate handle on the age/metallicity degeneracy.

Moreover, SBF magnitudes are required to be corrected for the dust extinction caused by our own Milky Way galaxy. Usually, the 100- $\mu \mathrm{m}$ DIRBE/IRAS dust emission maps by Schlegel, Finkbeiner \& Davis (1998) are adopted. In the future, improved galactic extinction values could be derived from multi-wavelength studies involving mid-IR or Spitzer/IRAC combined with blue ultraviolet (UV) GALEX observations.

The uncertainty in the zero-point of the Cepheid calibration is less than $0.1 \mathrm{mag}$ (see Section 4 above). However, the revised Cepheid calibration (Freedman et al. 2001) depends on the metallicity of the underlying stellar population and needs to be accounted for (Sakai et al. 2004; Macri et al. 2006; Scowcroft et al. 2009). This additional metallicity correction is of the order of -0.06 mag for the distance moduli and therefore adds only a small uncertainty of $0.08-0.10$ mag when measuring distances based on SBFs (Jensen et al. 2003; Blakeslee et al. 2010; see also Section 3.1).

\section{Theory meets Observation}

\subsection{Origin of $S B F S$}

Since the absolute SBF magnitude $\bar{M}$ in a given wavelength range depends on the underlying stellar population properties, SBF luminosities in different bandpasses probe different stages of stellar evolution within the unresolved stellar host system. UV and blue SBF magnitudes are sensitive to the evolution of stars within the hot horizontal branch (HB) and post-asymptotic giant branch (post-AGB) phases; optical SBF magnitudes serve as a measure of stars within the RGB and HB ranges, whereas NIR SBF luminosities represent a stellar evolution stage within the AGB and TP-AGB phases. Since the SBF luminosity is weighted by the square of the stellar luminosity (see Equation 2), it is extremely sensitive to the most luminous giant stars. Figure 7 illustrates the higher sensitivity of the SBF signal to luminous cool giant stars in their late stellar evolutionary stages compared with the total integrated stellar flux signal. The stellar evolutionary phases are ordered according to their relative contribution to the total integrated light and range from the zero-age main sequence (ZAMS) to the late AGB phase. For the computation a 12-Gyr old, solarmetallicity, single-burst stellar population model using the Padova evolutionary tracks with semi-empirical spectral energy distributions (SEDs) was adopted (Marigo et al. 2008). It can be seen that the integrated flux arises from stars throughout all stellar evolutionary 

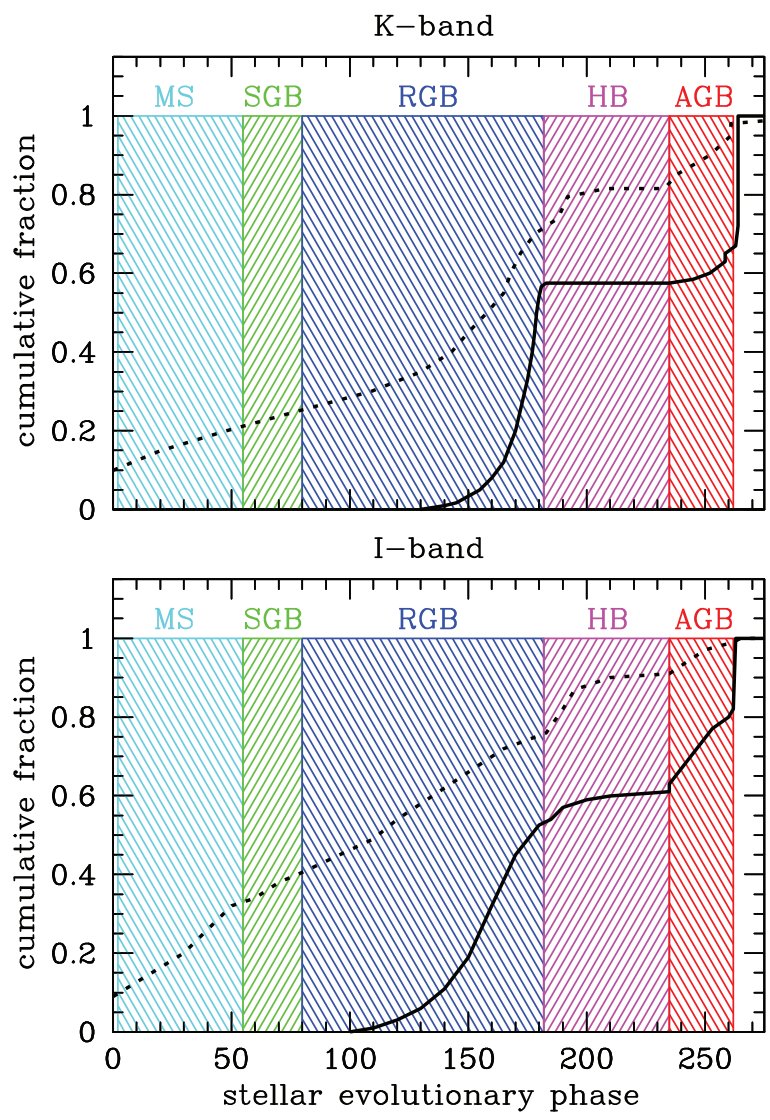

Figure 7 Origin of the SBF signal. For an old, solar-metallicity, single-burst stellar population model (see text) the fraction of the total light is given as a function of stellar evolutionary phase. Evolutionary phases range from the ZAMS (left) to the late AGB phase (right) in arbitrary units. The integrated flux (dotted line) arises from all stars of all stellar evolutionary phases. In contrast, the IR SBF signal (solid line, top panel) originates entirely from the RGB and AGB phases, with some additional contribution from the HB for optical SBFs (solid line, lower panel).

phases. In contrast, the IR SBF signal originates entirely from the RGB and AGB phases ( $\sim 88 \%$ of the flux is within the two brightest IR magnitudes), whereas for optical SBFs some additional contribution comes from stars along the HB $(\sim 60 \%$ of the signal is concentrated within the $3-4$ brightest optical magnitudes).

From Figure 7 it becomes evident that the SBF luminosities and colours should closely trace the peak luminosity and colours of late cool stars along the giant branch. Therefore, in the following section we give a brief summary of the main evolutionary phases of cool giant stars, the RGB phase (H-shell burning) and the AGB phase (He-shell burning).

\subsection{The Late Stages of Cool Giant Stars}

Low- and intermediate-mass stars of the main sequence $\left(1 \leq M / \mathrm{M}_{\odot} \leq 8\right)$ experience high luminosities as red giants in their late evolutionary phase along the AGB (up to a few $\left.10^{4} L / L_{\odot}\right)$. However, due to their low effective temperatures $\left(T_{\text {eff }} \leq 3500 \mathrm{~K}\right)$, these AGB stars are highly extended objects with radii and cool atmospheres of

several $100 R / R_{\odot}$. Depending on the initial ZAMS mass of the star, the hydrogen $(\mathrm{H})$ in the stellar core will be exhausted, and subsequently the helium $(\mathrm{He})$ core will rapidly contract and heat up, while the $\mathrm{H}$ will continue to burn in a shell around the nucleus. This stage marks the start of the RGB phase (H-shell burning). The beginning of the AGB phase is characterized by the end of $\mathrm{He}$ burning in the stellar core. In the first phase, the $\mathrm{He}$ burning continues in a shell around the core (early AGB). On the upper part of the AGB, the star becomes unstable because of strong radial pulsations with typical timescales of a few tens to hundreds of days (e.g. Iben \& Renzini 1983; Vassiliadis \& Wood 1993; Dorfi \& Hoefner 1998). The star enters the TP-AGB phase of alternate $\mathrm{H}$ - and $\mathrm{He}$-shell burning. In this phase, the AGB star shows thermal pulses: the H-shell burning is interrupted at regular times by the ignition of the He that has accumulated under the $\mathrm{H}$ shell beforehand as a by-product of $\mathrm{H}$ burning. Further, the variable convection zones mix material processed in the nucleus to the stellar surface, thereby changing the chemical composition of the convective layers, i.e. dredge-up phases (Iben \& Renzini 1983). Heavier elements (mainly carbon, nitrogen, oxygen, and s-process elements) are ultimately transported outwards into the stellar photosphere and modify the stellar surface composition. The relative fraction of carbon to oxygen $([\mathrm{C} / \mathrm{O}]$ ratio) is defined by the core mass and this mixing ratio regulates the generation of different molecules in the cool stellar atmosphere (e.g. TiO, C2, $\mathrm{CN}$ ). Most frequent are oxygen-rich AGB stars (M star: $[\mathrm{C} / \mathrm{O}]<0.95$ ), but sometimes a carbon-rich star $([\mathrm{C} / \mathrm{O}]>1.0)$ is formed, which eventually develops into a central white dwarf star within a planetary nebula.

Since the SBF signal is more sensitive to the most luminous stars in a stellar system, the SBFs provide tighter constraints on the evolution of evolved cool giant stars in elliptical galaxies than integrated luminosities alone. However, several aspects of the physics of cool giant stars (e.g. interior structure, mass-loss, mixing length for internal convection, pulsation modes), as well as their exact spectral energy distributions, remain poorly understood. Ideal observational targets would be nearby GCs, which host these stellar populations of homogenous composition and age. However, because cool giants cross their late evolutionary phase rapidly, only a handful of these stars exist at the present time for a given cluster. To overcome uncertainties in GC analyses such as smallnumber statistics or cosmic variance, SBF measurements of individual galaxies can represent an alternative approach, as the SBF signal is dominated by much higher luminosities and the stellar systems are expected to comprise metal-rich populations.

Since a few years, more attention has been dedicated in observing young intermediate-age stellar populations $(0.5 \lesssim \mathrm{Gyr} \lesssim 3)$ in GCs and stellar clusters in the MCs (see e.g. González-Lópezlira et al. 2005; Raimondo et al. 2005; Mouhcine, González \& Liu 2005; Lee et al. 2010). The brightest cluster stars are carbon-type AGB stars with 
bolometric luminosities that exceed the brightness of the tip of the RGB. In contrast to M-type AGB stars, carbon AGB stars are more luminous and also redder.

Therefore, IR SBFs represent a promising tool to disentangle the effects and contributions of ages and metallicities in unresolved stellar systems. In particular, mid-infrared (MIR) SBFs in the $L(3.5 \mu \mathrm{m})$ and $M$ bands $(4.8 \mu \mathrm{m})$ are expected to be highly sensitive tracers of the age of a stellar population. Because of the extremely high thermal IR background of the Earth's atmosphere, however, observations from the ground are only possible for the very nearest and bright galaxies (e.g. M31). In the foreseeable future, a new window on MIR SBFs will be opened with the James Webb Space Telescope (JWST), which will access both the $L$ and $M$ bands.

\subsection{Improved Stellar Population Models}

Today the whole extragalactic community is aware that stars in their TP-AGB phase are responsible for more than half of the NIR flux that originates from a stellar population at intermediate ages (several $100 \mathrm{Myr}$ to 1-2 Gyr). In some cases, the TP-AGB contribution may even increase to $80 \%$, depending on the range of ages and metallicities adopted in the modelling (Maraston 2005). As a consequence, AGB stars are the dominant contributors to the NIR mass-to-light ratio of intermediateage stellar populations (see Section 5.2), which are particularly sensitive for stellar mass estimates of highredshift galaxies at $z \sim 2$ (Maraston et al. 2006).

Over the past few years, significant progress has been made in our theoretical understanding of stellar populations (Biscardi et al. 2008; Raimondo 2009; Conroy, Gunn \& White 2009; Lee et al. 2010; González-Lópezlira et al. 2010; Conroy \& Gunn 2010). Substantial improvements were obtained regarding the uncertain and unknown late stages in the evolution of massive, evolved stars, in particular the (post)-AGB phase and the horizontal branch phase. A full discussion is beyond the scope of this review. However, one of the most promising techniques using the flexible synthesis of stellar populations, which has been verified through detailed comparisons with high-quality observations, is presented in the following.

Flexible stellar population synthesis (FSPS) descriptions take a novel approach in the construction of synthetic SSP spectra (Conroy et al. 2009; Conroy \& Gunn 2010). Through a parameterization of uncertain stages in the stellar evolution and by allowing these variables to alter freely, both the theoretically motivated galaxy properties and different sets of observational constraints can be modelled in combination. In particular, FSPS allows researchers to compute SSPs for a range of initial mass functions (IMFs) and metallicities and for a variety of assumptions regarding the morphology of the horizontal branch, the blue straggler population, the post-AGB phase, and the location in the Hertzsprung-Russell diagram of the TP-AGB phase. From these SSPs, composite stellar populations (CSPs) for a variety of star-formation histories (SFHs) and dust attenuation prescriptions can be generated. Furthermore, the descriptions offer different choices of isochrones and stellar libraries and provide weights to various stages of stellar evolution.

Conroy et al. point out that the unmodified Padova models result in a substantial disagreement with the observed properties of stellar clusters. This disagreement is amplified to a significant extent by a large population of luminous carbon stars as predicted by the models. Therefore, the bolometric luminosity $\left(L_{\mathrm{bol}}\right)$ along the TP-AGB phase was substantially lowered by accounting for shifts in the effective temperature $T_{\text {eff }}\left(\Delta_{T}\right)$ and $L_{\text {bol }}\left(\Delta_{L}\right)$ along the entire TP-AGB phase (Conroy et al. 2009). These applied changes are independent of metallicity and reduce the importance and dominance (both the overall number and higher number of more luminous O-rich stars) for all TP-AGB stars, including carbon stars. The resulting modified Padova isochrones are in much better agreement with both MC star clusters and post-starburst galaxies (see Figure 8). The substantial disagreement between the unmodified Padova calculations and the star cluster data arises from three differences: the usage of old inferiorquality data, the application of theoretical atmosphere models with dust radiative transfer descriptions (Loidl, Lançon \& Jørgensen 2001), and the application of the LF of carbon stars found in the MCs. In particular, the carbon star models are significantly bluer $(>1 \mathrm{mag}$ in $V-K)$ than observed carbon stars (R. Gautschy 2009, private communication). Further, the new models by Conroy \& Gunn (2010) adopt observed stellar spectra including the TP-AGB phase, which provide a better description of the observed properties of O-rich and carbon stars than synthetic modelled spectra.

In Figure 8, a comparison between FSPS models with both the Bag of Stellar Tracks and Isochrones (BaSTI) and modified Padova isochrones (Conroy \& Gunn 2010) and NIR SBF measurements within the $K$ band versus integrated $(V-I)_{0}$ colour plane is given. Further, data for star clusters in the MCs (González-Lópezlira et al. 2005) and SBF data for elliptical galaxies (Liu et al. 2002; Jensen et al. 2003) are included. The left-hand panel shows the results for the modified Padova isochrones, whereas the right-hand panel displays the findings for the modified FSPS + BaSTI models. It is clearly evident that the new modified FSPS + BaSTI prescriptions define a much broader range in the magnitude-colour plane than the unmodified predictions. In particular, the range of constant ages is more pronounced in the FSPS predictions and extends to the far blue colours of stellar MC clusters. Nevertheless, a subsample of galaxies comprises fainter SBF magnitudes and therefore is still poorly represented in both the BaSTI and Padova calculations. Because of the inverse relationship between NIR SBF luminosities and (stellar) masses, if SBF luminosities are overestimated as in the current model predictions then the derived stellar masses and mass-to-light ratios will be underestimated. 

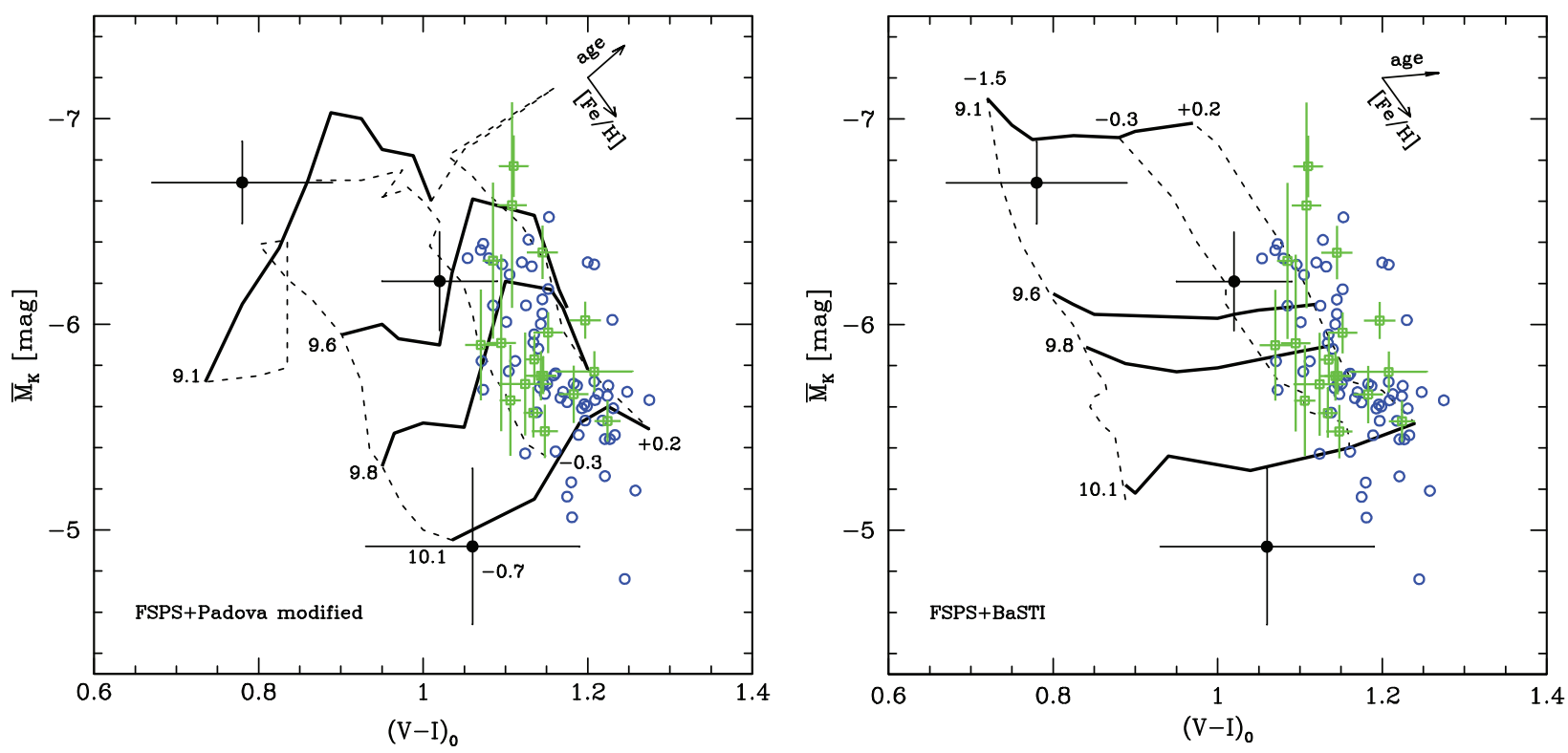

Figure $8 K$-band SBF fluctuation magnitude measurements and FSPS models as a function of integrated $(V-I)_{0}$ colour. Different FSPS model predictions (Conroy \& Gunn 2010, lines) are compared with observations of elliptical galaxies in the Fornax cluster (Liu et al. 2002, green squares), ellipticals in a variety of environments (Jensen et al. 2003, blue circles), and star clusters in the MCs (González-Lópezlira et al. 2005, solid black circles). For clarity reasons, uncertainties are displayed only for the Fornax galaxies and MC clusters. Model predictions are shown as lines of constant age (solid lines; in units of $\log (t / \mathrm{yr})$ ) and lines of constant metallicity (dashed lines; in units of $\log \left(Z / \mathrm{Z}_{\odot}\right)$ ). Arrows denote the direction of 0.02 and 0.05 dex variations in age and metallicity, respectively. FSPS models are constructed with the modified Padova isochrones (left panel) and the BaSTI isochrones (right panel). The unmodified Padova isochrones used in the past give only a poor approximation of half of the data. Large metallicity variations of $\Delta \log \left(Z / \mathrm{Z}_{\odot}\right) \sim 0.60$ dex can be seen, depending on the treatment of convection (convective core overshooting) and the TP-AGB phase in the stellar evolution.

Many observations of elliptical galaxies provide strong evidence for an $\alpha$ enhancement of heavy elements in their stellar populations (e.g. Worthey, Faber \& Gonzalez 1992; Ziegler et al. 2005). For the theoretical prescriptions in Figure 8, solar-scaled chemical compositions were assumed. However, recent models including an accurate description of the (TP)-AGB phase suggest that the level of $\alpha$ enhancement has a minor effect on the model predictions in the $(V-I)$ versus $\mathrm{F} 160 \mathrm{~W}$ SBF coordinate space (Lee et al. 2010). Nevertheless, the impact of other enrichments on the SBF luminosities and galaxy colours has not yet been explored in detail.

Overall, the new modifications and prescriptions such as FSPS offer a much better representation of the $K$-band SBF luminosities and colours, thereby addressing several limitations in the unmodified models (Conroy \& Gunn 2010): BaSTI isochrones predict too-blue SBF colours compared with the observations (implying too-hot AGB and TP-AGB temperatures), whereas the Padova isochrones provide too-bright and too-red SBF magnitudes and colours (which originate from an overabundance of carbon stars and an overluminous TP-AGB evolutionary phase).

So far, the current status of the theoretical modelling of SBF quantities provides some constraints on the average metallicities of unresolved stellar populations. For the future, a better understanding of the whole range of stellar evolution phases is crucial to gain detailed insight into the stellar population properties of globular clusters and galaxies.

\section{A Measure of Distance-Independent Luminosities}

Measuring absolute luminosities of galaxies that are distance-independent to a high precision is important for a wide range of astronomical applications (e.g. sizes of galaxies, black hole masses, total galaxy mass measurements). Tonry and collaborators have discovered a further parameterization of the SBF method (T01). Moreover, this description is not only distance-independent but also independent of photometric calibration or dust extinction. The parameter $\bar{N}$ is defined as the ratio of the total apparent flux from a galaxy to the flux provided by the fluctuation signal. In terms of magnitudes, this (absolute luminosity) measure is the difference between the fluctuation magnitude $\bar{m}$ and the total magnitude of the galaxy $\bar{m}_{\text {tot }}$, which corresponds to the total luminosity of the galaxy in units of the luminosity of a typical giant star within that galaxy as

$$
\bar{N}=\bar{m}-\bar{m}_{\mathrm{tot}}=+2.5 \log \left(\frac{L_{\mathrm{tot}}}{\bar{L}}\right) .
$$

$\bar{N}$ is also referred to as the 'fluctuation star count'.

As expected, galaxy colour correlates with $\bar{N}$ (which is a proxy for the absolute luminosity) following the relation

$$
(V-I)_{0}=0.317+0.042 \bar{N} .
$$

Figure 9 shows the dependence of the $(V-I)$ galaxy colour on the fluctuation star count $\bar{N}$. The correlation is 


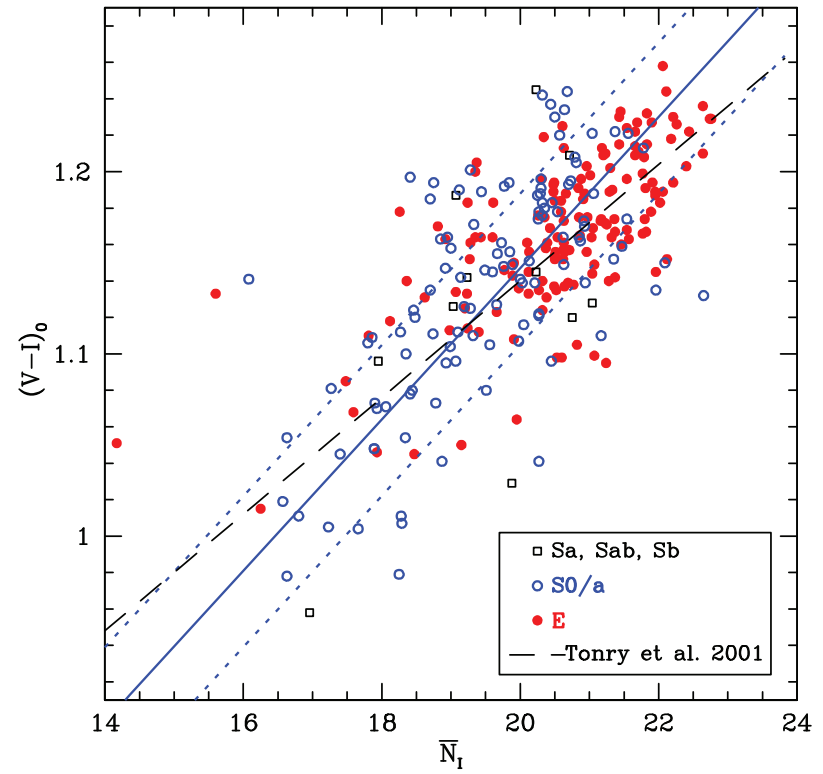

Figure $9(V-I)_{0}$ galaxy colour as a function of the fluctuation star count $\bar{N}_{I}$ for different morphological types (Blakeslee et al. 2001b). Symbols are as in Figure 4. The dashed line is the empirical relation from Tonry et al. (2001).

well established for different morphological types (E, S0, $\mathrm{Sa}$ ) and across a range of luminosities. The relation in Equation 11, which is shown in Figure 9 as the solid and dotted ( $\pm 1 \sigma$ error) lines, is slightly steeper but in good agreement with the correlation of $\bar{N}$ and $(V-I)$ found by T01 (dashed line).

Surprisingly, the slope of this relation is very shallow with an observed scatter in $(V-I)$ of 0.04 mag. Therefore, a large error in $\bar{N}$ translates into a negligible effect in $(V-I)$; for example, assuming $\delta \bar{N}=0.5 \mathrm{mag}$ corresponds to only $\delta(V-I)=0.016$ mag (T01). According to the prediction, the intrinsic scatter of the relation might be as small as $0.025 \mathrm{mag}$, which also suggests it is an efficient way of deriving accurate extinction measurements with rms uncertainties of $2 \%$.

Although there is some covariance from the application of $\bar{m}_{I}$ for both the distance modulus and the intrinsic galaxy colour, the actual covariance is very mild because of the shallow slope of the $\bar{N}-(V-I)$ relationship. Instead, using the galaxy colour to estimate $\bar{M}_{I}$ directly is challenging due to the observational requirements of high-precision photometry and the accurate assessment of the presence of and sensitivity to dust extinction.

Based on the I-band SBF survey, T01 found an empirical relationship between $\bar{M}_{I}$ and $\bar{N}$ following

$$
\bar{M}_{I}=1.74+0.14(\bar{N}-20) .
$$

Although this introduces a covariance between $\bar{M}_{I}$ derived in this way and $\bar{m}_{I}$, the resulting distance modulus is $14 \%$ less sensitive to (systematic) uncertainties in $\bar{m}_{I}$ (Blakeslee et al. 2001b). However, $\bar{N}$ should be not regarded as a substitute for $(V-I)$ when deriving $\bar{M}_{I}$, as the parameter is still relatively unexplored. Nevertheless, in the absence of a galaxy colour $\bar{N}$ provides an alternative way to measure a distance. For example, Cantiello, Brocato \& Capaccioli (2011) have adopted a calibration based on $\bar{N}$ to derive the distance to 12 nearby galaxies with an accuracy of about $30 \%$, where colour information was unavailable in the data archive. The consequence of the relationship of $\bar{N}$ with $\bar{M}_{I}$ is suggested to rely on the Fundamental Plane of early-type galaxies (Djorgovski \& Davis 1987; Dressler et al. 1987) and their projections (e.g. Fritz et al. 2005; 2009a). If a colour measurement is available, $\bar{N}$ should not supplant the application of $(V-I)$ to calibrate $\bar{M}_{I}$. Nevertheless, it appears that $\bar{N}$ offers a valuable alternative to measure a fairly reliable distance, but more work is needed to understand the parameter and its relatively large scatter fully.

\section{Conclusions}

SBFs are most likely the most efficient technique to measure the distance to unresolved nearby stellar systems. In terms of accuracy, depth, and completeness, the SBF technique represents a unique way of measuring extragalactic distances to early-type galaxies and spiral bulges. Moreover, as SBFs are an intrinsic property of an integrated stellar population, they give insight into and allow scientists to set constraints on the stellar populations and chemistry of globular clusters and early-type galaxies. Precise extragalactic distances are still extremely important and fundamental for various applications in astrophysics. High-accuracy distance measurements are essential because errors in distance usually translate into large uncertainties in the derived physical properties of individual galaxies, such as absolute luminosities, linear sizes, star formation rates and time-scales, black hole masses, and (total) masses of galaxies, as well as dark matter halo mass estimates. Moreover, for the nearby Universe, accurate distances serve as a reliable and trustworthy substitute for redshift as a distance indicator and provide the possibility of mapping the threedimensional structure and velocity field of the Local Volume.

Multi-wavelength SBF studies involving optical and NIR observations offer a unique way of analysing the properties and content of composite stellar populations, as different photometric bandpasses are sensitive to different phases of stellar evolution. In particular, stellar population gradients based on SBF measurements allow one to probe the merger and assembly history of nearby galaxies and unresolved stellar systems. An interesting by-product of detailed SBF data are possible constraints on the stellar populations and properties of globular cluster systems, such as understanding the nature and origin of the bi/multi-modal globular cluster colour distributions in giant elliptical galaxies, which remains an unsolved puzzle (Conroy \& Spergel 2011).

Apart from ground-based observations, a complete optical SBF survey from space will allow us to construct a complete map of the individual velocity patterns of 
galaxies and galaxy clusters down to an accuracy in distances where the bulk motions become just a few per cent $(\lesssim 3 \%)$ of the Hubble flow (see Section 3.1 for details). Based on WFC3/IR observations of 600 Cepheids to calibrate the magnitude-redshift relation of $253 \mathrm{SNe}$ Ia, the most recent measurement of the Hubble constant was derived down to an accuracy of $3 \%: H_{0}=73.8 \pm 2.4$ (stat) \pm 3.4 (sys) $\mathrm{km} \mathrm{s}^{-1} \mathrm{Mpc}^{-1}$ (Riess et al. 2011). Future observations will be able to measure the Hubble constant to a precision of $2 \%$ using the most accurate distance indicators. The extension of SBF studies with WFC3/IR will be a highly valuable asset from this perspective, both for stellar content and for precise mass estimates.

The combination of a precise direct measurement of the Hubble constant $H_{0}$ with constraints from the CMB provides a powerful test of different models of dark energy ( $\mathrm{Hu}$ 2005). In particular, the two independent approaches together allow the investigation of the evolution of cosmic energy density $w$ to $10 \%$ accuracy across a broad cosmic epoch of $0<z<1100$ into the early Universe of the 'dark ages' ( $\sim 13.5 \mathrm{Gyr})$. However, $w$ is highly susceptible to uncertainties in the value of $H_{0}$, with errors in $w$ being twice the fractional errors in $H_{0}$, thereby keeping $w$ and the flatness constant (Weinberg et al. 2012). The real challenge for future studies is therefore an accurate measurement of the local $H_{0}$ value to $1 \%$ precision, encompassing both statistical (random) errors and systematic uncertainties.

The drawback of an individual $H_{0}$ value is the restriction to a single number at a fixed redshift, so that only one particular model of dark energy can be tested but not possible deviations from the theoretical predictions. A high-precision measurement of $H_{0}$, however, significantly increases the power of other dark energy constraints by up to $40 \%$ (Weinberg et al. 2012). Moreover, a direct $H_{0}$ determination has the potential to uncover possible departures from the smooth evolution of dark energy at low redshift and could settle the controversy if the Universe is still in a phase of acceleration.

\section{Future Directions}

There is no doubt that the future of SBF lies in a combination of space-based and adaptive optics (AO) observations. The SBF fluctuation signal in the $H$ band is a factor of 30 larger than that in the $I$ band. Equally important, for $H S T /$ NICMOS observations the sky background is more than a factor of 100 lower compared with IR observations with ground-based telescopes. The HST WFC3/IR channel offers a unique tool with high spatial resolution $(\mathrm{FWHM}=0.13 \mathrm{arcsec})$, wide field of view $(2.05 \times 2.26$ $\operatorname{arcmin}^{2}$ ), good set of filters for SBF measurements, and good detector characteristics (e.g. sensitivity, noise, cosmetics). WFC3/IR observations in F110W $(1.25 \mu \mathrm{m}, \approx J)$ and $\mathrm{F} 160 \mathrm{~W}(1.6 \mu \mathrm{m}, \approx H)$ as well as ACS $z^{\prime}$-band $(0.85 \mu \mathrm{m})$ measurements will be useful complements to existing (ground-based) studies.
The most promising approach is a complete spacebased SBF IR survey to map out the nearby Universe. Such a project would allow scientists to constrain the stellar population and mass content of 'dynamically hot' galaxies and act as a powerful complement to ongoing surveys studying the properties of nearby galaxies in detail, such as SAURON (de Zeeuw et al. 2002) or ATLAS $^{3 \mathrm{D}}$ (Cappellari et al. 2011). ${ }^{8}$ A crucial complement to space observations is offered by AO observations with NIR instruments [e.g. ALTtitude conjugate Adaptive optics for the InfraRed (ALTAIR), OH-Suppressing InfraRed Integral-field Spectrograph (OSIRIS), Nasmyth Adaptive Optics System Near-Infrared Imager and Spectrograph (NACO), InfraRed Camera and Spectrograph (IRCS)] with excellent seeing conditions between $\lesssim 0.03-0.05$ arcsec FWHM in the IR wavelength regime, feasible with current state-of-the-art 8-m class telescopes such as Gemini, Keck, VLT, or Subaru. Future 30-m class telescopes such as the European Extremely Large Telescope $\left(\right.$ E-ELT) ${ }^{9}$ or Thirty Meter Telescope (TMT) ${ }^{10}$ will be operated consecutively using AO instruments.

Multi-band SBF studies across a broad wavelength regime will be the ultimate key to understanding the stellar populations of stellar systems and their properties. If it is possible to conceive completely the dependence of $\bar{M}$ across the full wavelength spectrum, the SBF method will then be independent of the calibration of the primary distance indicators of Cepheids.

The future JWST will open a new window for SBFs in the MIR, which is currently prohibitively expensive and challenging from the perspective of ground-based observations (telescope exposure time and uncertainties in the bright sky background). Promising wavelength ranges are the $L(3.5 \mu \mathrm{m})$ and $M(4.8 \mu \mathrm{m})$ bands, as both are expected to be highly sensitive to the age content of old, evolved stellar populations. In particular, the MIR SBFs will allow astronomers to constrain the mass-loss rates of AGB stars and pin down whether there is a relation between the mass loss and metallicity of a stellar population as expected from stellar synthesis modelling (González-Lópezlira et al. 2010).

Moreover, the Gaia astrometry satellite, ${ }^{11}$ expected to launch in May 2013, will have a dramatic impact through a re-definition of the cosmic distance scale. Proper motions for stars and new trigonometric parallaxes of 10,000 (partly long-period) Cepheids will be measured within $5 \mathrm{kpc}$; hence geometric distances of the most powerful 'primary' distance indicators (e.g. Cepheids, RR Lyrae stars, statistical/trigonometric parallaxes) will be improved with unprecedented accuracy (between 10$100 \mu \operatorname{arcsec}, \sim 25 \mu \operatorname{arcsec}$ at $V=15 \mathrm{mag}$ ). Distances with

\footnotetext{
${ }^{8}$ ATLAS $^{3 \mathrm{D}}$ : http: / / www-astro.physics.ox.ac.uk/atlas3d

${ }^{9}$ E-ELT: http: / /www. eso.org/public/teles-instr/e-elt

${ }^{10}$ TMT: http : / /www . tmt . org

${ }^{11}$ Gaia satellite: http: //gaia.esa.int/
} 
precision at sub-percentage-level will pave the way for a direct refinement of the calibration of 'secondary' distance indicators (e.g. SBF, SNe Ia/II, novae, $D_{n}-\sigma$, Tully-Fisher relation) and a global re-assessment of the entire cosmic distance ladder (see also Section 1.2). Moreover, due to the frequent repeated observations of the same areas on the sky, Gaia will also detect and locate transient events, such as gamma-ray bursts (GRBs), binaries and $\mathrm{SNe}$ with a completeness down to $G=20$ mag, to high accuracy.

The combination of both high-resolution trigonometric parallaxes and NIR-AO observations will lift the SBF technique to the next level, establish an ultimate reference point for the cosmic distance scale, and settle the controversy regarding the local Hubble flow (Lynden-Bell et al. 1988; Tonry et al. 2000). The future of the SBF method still shines extremely brightly.

\section{Acknowledgments}

The author is grateful to Professor Joseph B. Jensen for stimulating discussions and many valuable comments and suggestions on an earlier version of the draft. The anonymous referee is thanked for a constructive review, which improved the clarity of the manuscript. The author acknowledges support from a VIPERS Fellowship through a PRIN-INAF 2008 grant (VIPERS) and partial support from grant HST-GO-10826.01 from the Space Telescope Science Institute, which is operated by the Association of Universities for Research in Astronomy, Inc., under NASA contract NAS 5-26555.

\section{References}

Aaronson, M., et al., 1989, ApJ, 338, 654

Ajhar, E. A. \& Tonry, J. L., 1994, ApJ, 429, 557

Ajhar, E. A., et al., 1997, AJ, 114, 626

Ajhar, E. A., et al., 2001, ApJ, 559, 584

Baade, W., 1944, ApJ, 100, 137

Barber DeGraaff, R., Blakeslee, J. P., Meurer, G. R. \& Putman, M. E., 2007, ApJ, 671, 1624

Baum, W., 1986, STcI GTO proposal 1114-GTO/WFP

Baum, W. A., 1990, in ASP Conf. Ser. 10, Evolution of the Universe of Galaxies, ed. R. G. Kron (San Francisco: ASP), 119.

Baum, W. A. \& Schwarzschild, M., 1955, AJ, 60, 247

Biscardi, I., Raimondo, G., Cantiello, M. \& Brocato, E., 2008, ApJ, 678, 168

Blakeslee, J. P. \& Tonry, J. L., 1995, ApJ, 442, 579

Blakeslee, J. P., Ajhar, E. A. \& Tonry, J. L., 1999, in Post-Hipparcos Cosmic Candles, ed. A. Heck \& F. Caputo (Dordrecht: Kluwer), 181

Blakeslee, J. P., et al., 2001a, MNRAS, 320, 193

Blakeslee, J. P., Lucey, J. R., Barris, B. J., Hudson, M. J. \& Tonry, J. L., 2001b, MNRAS, 327, 1004

Blakeslee, J. P., Lucey, J. R., Tonry, J. L., Hudson, M. J. \& Narayanan, V. K., 2002, MNRAS, 330, 443

Blakeslee, J. P., et al., 2009, ApJ, 694, 556

Blakeslee, J. P., et al., 2010, ApJ, 724, 657

Cantiello, M., Blakeslee, J. P., Raimondo, G., Mei, S., Brocato, E. \& Capaccioli, M., 2005, ApJ, 634, 239

Cantiello, M., Blakeslee, J. P., Raimondo, G., Brocato, E. \& Capaccioli, M., 2007a, ApJ, 668, 130

Cantiello, M., Raimondo, G., Blakeslee, J. P., Brocato, E. \& Capaccioli, M., 2007b, ApJ, 662, 940
Cantiello, M., Brocato, E. \& Capaccioli, M., 2011, A\&A, 534, A35 Cappellari, M., et al., 2011, MNRAS, 413, 813

Ciardullo, R., 2006, in Planetary Nebulae beyond the Milky Way, ed. L. Stanghellini, J. R. Walsh \& N. G. Douglas (Berlin: Springer), 79

Conroy, C. \& Gunn, J. E., 2010, ApJ, 712, 833

Conroy, C. \& Spergel, D. N., 2011, ApJ, 726, 36

Conroy, C., Gunn, J. E. \& White, M., 2009, ApJ, 699, 486

de Vaucouleurs, G., 1961, ApJS, 5, 233

de Zeeuw, P. T., et al., 2002, MNRAS, 329, 513

Djorgovski, S. \& Davis, M., 1987, ApJ, 313, 59

Dorfi, E. A. \& Hoefner, S., 1998, in Rev. Modern Astron. 11, Stars and Galaxies, ed. R. E. Schielicke (Hamburg: Astronomische Gesellschaft), 147

Dressler, A., Lynden-Bell, D., Burstein, D., Davies, R. L., Faber, S. M., Terlevich, R. \& Wegner, G., 1987, ApJ, 313, 42

Dunn, L. P. \& Jerjen, H., 2006, AJ, 132, 1384

Freedman, W. L., 1989, AJ, 98, 1285

Freedman, W. L. \& Madore, B. F., 2010, ARA\&A, 48, 673

Freedman, W. L., et al., 2001, ApJ, 553, 47

Fritz, A., 2000, Diploma Thesis, University of Vienna

Fritz, A., 2002, BaltA, 11, 385

Fritz, A., Ziegler, B. L., Bower, R. G., Smail, I. \& Davies, R. L., 2005, MNRAS, 358, 233

Fritz, A., Böhm, A. \& Ziegler, B. L., 2009a, MNRAS, 393, 1467

Fritz, A., Jørgensen, I., Schiavon, R. P. \& Chiboucas, K., 2009b, AN, 330, 931

Giovanelli R., et al., 1997, AJ, 113, 53

González-Lópezlira, R. A., Albarrán, M. Y., Mouhcine, M., Liu, M. C., Bruzual, A. G. \& de Batz, B., 2005, MNRAS, 363, 1279 González-Lópezlira, R. A., et al., 2010, MNRAS, 403, 1213

Hu, W., 2005, in ASP Conf. Ser. 339, Observing Dark Energy, ed. S. C. Wolff \& T. R. Lauer (San Francisco: ASP), 215

Hubble, E. P., 1926, ApJ, 63, 236

Hubble, E., 1929, PNAS, 15, 168

Hubble, E. \& Humason, M. L., 1931, ApJ, 74, 43

Iben, I., Jr \& Renzini, A., 1983, ARA\&A, 21, 271

Jacoby, G. H., et al., 1992, PASP, 104, 599

Jensen, J. B., Luppino, G. A. \& Tonry, J. L., 1996, ApJ, 468, 519

Jensen, J. B., Tonry, J. L. \& Luppino, G. A., 1998, ApJ, 505, 111

Jensen, J. B., Tonry, J. L. \& Luppino, G. A., 1999, ApJ, 510, 71

Jensen, J. B., et al., 2001, ApJ, 550, 503

Jensen, J. B., et al., 2003, ApJ, 583, 712

Jerjen, H., Rekola, R., Takalo, L., Coleman, M. \& Valtonen, M., 2001, A\&A, 380, 90

Jerjen, H., Binggeli, B. \& Barazza, F. D., 2004, AJ, 127, 771

Leavitt, H. S., 1908, AnHar, 60, 87

Lee, H.-c., Worthey, G. \& Blakeslee, J. P., 2010, ApJ, 710, 421

Lemaître, G., 1927, ASSB, 47, 49

Liu, M. C., Charlot, S. \& Graham, J. R., 2000, ApJ, 543, 644

Liu, M. C., Graham, J. R. \& Charlot, S., 2002, ApJ, 564, 216

Loidl, R., Lançon, A \& Jørgensen, U. G., 2001, A\&A, 371, 1065

Lorenz, H., Bohm, P., Capaccioli, M., Richter, G. M. \& Longo, G., 1993, A\&A, 277, L15

Luppino, G. A. \& Tonry, J. L., 1993, ApJ, 410, 81

Lynden-Bell, D., Faber, S. M., Burstein, D., Davies, R. L., Dressler, A., Terlevich, R. \& Wegner, G., 1988, ApJ, 326, 19

Macri, L. M., Stanek, K. Z., Bersier, D., Greenhill, L. J. \& Reid, M. J., 2006, ApJ, 652, 1133

Maraston, C., 2005, MNRAS, 362, 799

Maraston, C., et al., 2006, ApJ, 652, 85

Marigo, P., Girardi, L., Bressan, A., Groenewegen, M. A. T., Silva, L. \& Granato, G. L., 2008, A\&A, 482, 883

Mei, S., et al., 2001, A\&A, 366, 54

Mei, S., et al., 2005, ApJ, 625, 121

Mei, S., et al., 2007, ApJ, 655, 144

Mieske, S., Hilker, M. \& Infante, L., 2006, A\&A, 458, 1013

Mieske, S., Hilker, M., Infante, L. \& Mendes de Oliveira, C., 2007, A\&A, 463, 503 
Mouhcine, M., González, R. A. \& Liu, M. C., 2005, MNRAS, 362,1208

Mould, J., Kristian, J. \& Da Costa, G. S., 1983, ApJ, 270, 471

Mould, J., Kristian, J. \& Da Costa, G. S., 1984, ApJ, 278, 575

Mould, J. R., et al., 2000, ApJ, 529, 786

Pahre, M. A. \& Mould, J. R., 1994, ApJ, 433, 567

Pahre, M. A., et al., 1999, ApJ, 515, 79

Peletier, R. F., Davies, R. L., Illingworth, G. D., Davis, L. E. \& Cawson, M., 1990, AJ, 100, 1091

Pritchet, C. J. \& van den Bergh, S., 1988, ApJ, 331, 135

Raimondo, G., 2009, ApJ, 700, 1247

Raimondo, G., Brocato, E., Cantiello, M. \& Capaccioli, M., 2005, $\mathrm{AJ}, 130,2625$

Riess, A. G., et al., 2009, ApJ, 699, 539

Riess, A. G., et al., 2011, ApJ, 730, 119

Sakai, S., Ferrarese, L., Kennicutt, R. C., Jr \& Saha, A., 2004, ApJ, 608,42

Sandage, A. R. \& Tammann, G. A., 1982, ApJ, 256, 339

Schlegel, D. J., Finkbeiner, D. P. \& Davis, M., 1998, ApJ, 500, 525

Scowcroft, V., Bersier, D., Mould, J. R. \& Wood, P. R., 2009, MNRAS, 396, 1287

Shapley, H., 1919, ApJ, 50, 107
Shopbell, P. L., Bland-Hawthorn, J. \& Malin, D. F., 1993, AJ, 106,1344

Simard, L. \& Pritchet, C. J., 1994, ApJ, 107, 503

Sodemann, M. \& Thomsen, B., 1995, AJ, 110, 179

Sodemann, M. \& Thomsen, B., 1996, AJ, 111, 208

Tonry, J. L., 1991, ApJ, 373, L1

Tonry, J. L. \& Schneider, D. P., 1988, AJ, 96, 807

Tonry, J. L., Ajhar, E. A. \& Luppino, G. A., 1990, AJ, 100, 1416 (TAL90)

Tonry, J. L., Blakeslee, J. P., Ajhar, E. A. \& Dressler, A., 1997, ApJ, 475, 399

Tonry, J. L., Blakeslee, J. P., Ajhar, E. A. \& Dressler, A., 2000, ApJ, 530,625

Tonry, J. L., Dressler, A., Blakeslee, J. P., Ajhar, E. A., Fletcher, A. B., Luppino, G. A., Metzger, M. R. \& Moore, C. B., 2001, ApJ, 546, 681

Vassiliadis, E. \& Wood, P. R., 1993, ApJ, 413, 641

Weinberg, D. H., et al., 2012, preprint (arXiv:1201.2434)

Worthey, G., 1993, ApJ, 409, 530

Worthey, G., Faber, S. M. \& Gonzalez, J. J., 1992, ApJ, 398, 69

Ziegler, B. L., Thomas, D., Böhm, A., Bender, R., Fritz, A. \& Maraston, C., 2005, A\&A, 433, 519 